\title{
MFS fading regularization method for the identification of boundary conditions from partial elastic displacement field data
}

\author{
L. Cailléa, J-L. Hanus $\mathbb{D}^{\mathrm{b}}$, F. Delvare $\mathbb{D}^{\mathrm{a}}{ }^{\text {and N. Michaux-Leblond }}{ }^{\mathrm{a}}$
}

aUNICAEN, CNRS, LMNO, Normandie Univ, Caen, France; 'baboratoire de Mécanique Gabriel Lamé, INSA Centre Val de Loire, Université d'Orléans, Université de Tours, Bourges, France

\begin{abstract}
A method is proposed to solve an inverse problem in twodimensional linear isotropic elasticity. The inverse problem consists of the determination of both the entire displacement field and the boundary conditions inaccessible to the measurement from the partial knowledge of the displacement field. The algorithm is based on a fading regularization method (FRM) and is numerically implemented using the method of fundamental solutions (MFS). The inverse technique is first validated with synthetic data and is then applied to the interpretation of experimental measurements obtained by digital image correlation (DIC).
\end{abstract}

\section{ARTICLE HISTORY}

Received 22 December 2017 Accepted 14 December 2018

\section{KEYWORDS}

Inverse problems; regularization method; method of fundamental solutions; linear elasticity; partial full-field measurements

\section{Introduction}

Numerous mechanical problems can be considered as inverse problems in the sense that the geometry of the domain, the equilibrium equations, the constitutive equations, the boundary conditions on the whole boundary of the domain and potentially the initial conditions are not all given. According to this definition, many mechanical problems, for example, identification of material parameters, identification of unknown boundaries, identification of residual stresses, identification of inaccessible boundary conditions can be considered as inverse problems. Such inverse problems are in general ill-posed, in the sense that the existence, uniqueness and stability of their solutions are not always guaranteed, see e.g. Hadamard (Hadamard, 1923). There are numerous important contributions in the literature for inverse boundary problems in elasticity, which are characterized by incomplete boundary conditions. The lack of complete boundary conditions is usually overcome by supplying additional information in the form of either internal displacements, strain or stress measurements, or over-specified boundary conditions on the accessible 
part of the boundary. Many authors are interested in recovering the missing data on some part of the boundary of a domain from overspecified data on the other part. This problem is known as a Cauchy problem.

There are many regularization techniques, like Tikhonov-type regularization methods (Koya, Yeih, \& Mura, 1993, Maniatty, Zabaras, \& Stelson, 1989, Marin \& Lesnic, 2002, 2003, 2004, Tikhonov \& Arsenin, 1977, Yeih, Koya, \& Mura, 1993) and iterative methods (Andrieux \& Baranger, 2008, Baranger \& Andrieux, 2008, Marin, Elliott, Ingham, \& Lesnic, 2001, Marin, Hào, \& Lesnic, 2002, Marin \& Lesnic, 2005), used to obtain stable approximated solution of inverse boundary value problems in elasticity. Cimetière et al. (Cimetière, Delvare, Jaoua, \& Pons, 2001, Cimetière, Delvare, \& Pons, 2000) introduced the so-called fading regularization method to solve the Cauchy problem associated with the Laplace equation. This regularization procedure consists of an iterative fixed-point process. This approach reduces the resolution of the Cauchy problem to that of a sequence of optimization problems subject to an equality constraint.

At each step of this iterative procedure, Cimetière et al. (Cimetière et al., 2001, 2000) look, among all solutions of the equilibrium equations, for the one that fits at best the over-specified data and the previously computed optimal element. This is the reason why the functional, to be minimized in the space that characterizes the solutions of the equilibrium equations, consists of two terms: a relaxation term which allows to minimize the gap between the optimal element and the over-specified boundary data, and a regularization term which controls the gap between the actual optimal element and the previous one. In the case of compatible data, Cimetière et al. (Cimetière et al., 2001, 2000) proved that the sequence converges to the solution of the given Cauchy problem and that the additional regularization term tends to zero as the iterative procedure is continued. This is the reason why this method is named the fading regularization method (FRM).

The FRM was numerically implemented, in two dimensions, in the case of the Cauchy problem for the Laplace equation, using both the boundary element method (BEM) and the finite element method (FEM) by Delvare et al. (Delvare, Cimetière, \& Pons, 2002) and Cimetière et al. (Cimetière, Delvare, Jaoua, \& Pons, 2002), respectively. It was later extended to solve Cauchy problems in linear isotropic elasticity in conjunction with the FEM (Delvare, Cimetière, Hanus, \& Bailly, 2010, Durand, Delvare, \& Bailly, 2011) and the method of fundamental solutions (MFS) (Marin, Delvare, \& Cimetière, 2016). The FRM was also numerically implemented using the MFS to solve Cauchy problem for the Helmholtz equation (Caillé, Delvare, Marin, \& Michaux-Leblond, 2017). 
This paper represents a contribution to the study of less academic problems. Indeed, numerous inverse boundary value problems arise from the limits of experimental instrumentation. In experimental solid mechanics, due to the progress in the field of computer vision applied to experimental measurements, field measurements on a part of the specimen surface are now common. Among the different non-contact optical methods, the Digital Image Correlation technique, initially started in 1980s, has become increasingly popular, especially in 2D problems, to determine the displacement field (Grédiac \& Hild, 2012, Sutton, Orteu, \& Schreier, 2009). However, the complete measurement chain introduces an important number of error sources: the quality of the camera, regularity and uniformity of the lighting system, spatial contrast of the random speckle pattern, DIC algorithm, etc. The main consequence is that the displacement field must be considered as disturbed by noise and the inverse problem should be solved by limiting the influence of displacement errors on the identification results. For this reason, the inverse problem should be solved in a stable manner by preventing amplification of measurement errors (Dennis, Dulikravich, \& Yoshimura, 2004), especially when the amount of noise is significant (Arai, Nishida, \& Adachi, 2000).

This paper investigates such an inverse problem in linear isotropic elasticity. It consists, from the knowledge of both the displacement field measured in a restricted area of the domain and the stress vector on a part of the boundary, of reconstructing simultaneously the elastic displacement field in the whole domain and the boundary conditions. Due to the noise that affects measurements, this problem is ill-posed and is numerically solved, in a stable manner, by combining a FRM with a MFS numerical method. The paper is organized as follows. In Section 2 we present the inverse problem under investigation. The combination of a fading regularization technique and of the MFS numerical method is described in Section 3. In Section 4, the proposed method is applied to two examples with synthetic numerical data. Then, in Section 5, the proposed method is applied to an experimental diametral compression where the partial displacements data are obtained from experimentations using the Digital Image Correlation technique and therefore are noisy. Finally, some concluding remarks and ideas for future works are presented in Section 6.

\section{Inverse problem formulation}

We consider an isotropic linear elastic material which occupies an open bounded domain $\Omega \subset \mathbb{R}^{2}$ and assume that $\Omega$ is bounded by a piecewise smooth boundary $\Gamma \equiv \partial \Omega$, such that $\Gamma=\Gamma_{d} \cup \Gamma_{u}$, where $\Gamma_{d}, \Gamma_{u} \neq \emptyset$ and $\Gamma_{d} \cap \Gamma_{u}=\emptyset$. We also define $\Omega_{d}$ which is a part of $\Omega\left(\Omega_{d} \subset \Omega\right.$ and $\Omega_{d} \neq \Omega$ ) (Figure 1).

In the absence of body forces, the equilibrium equations are given by 


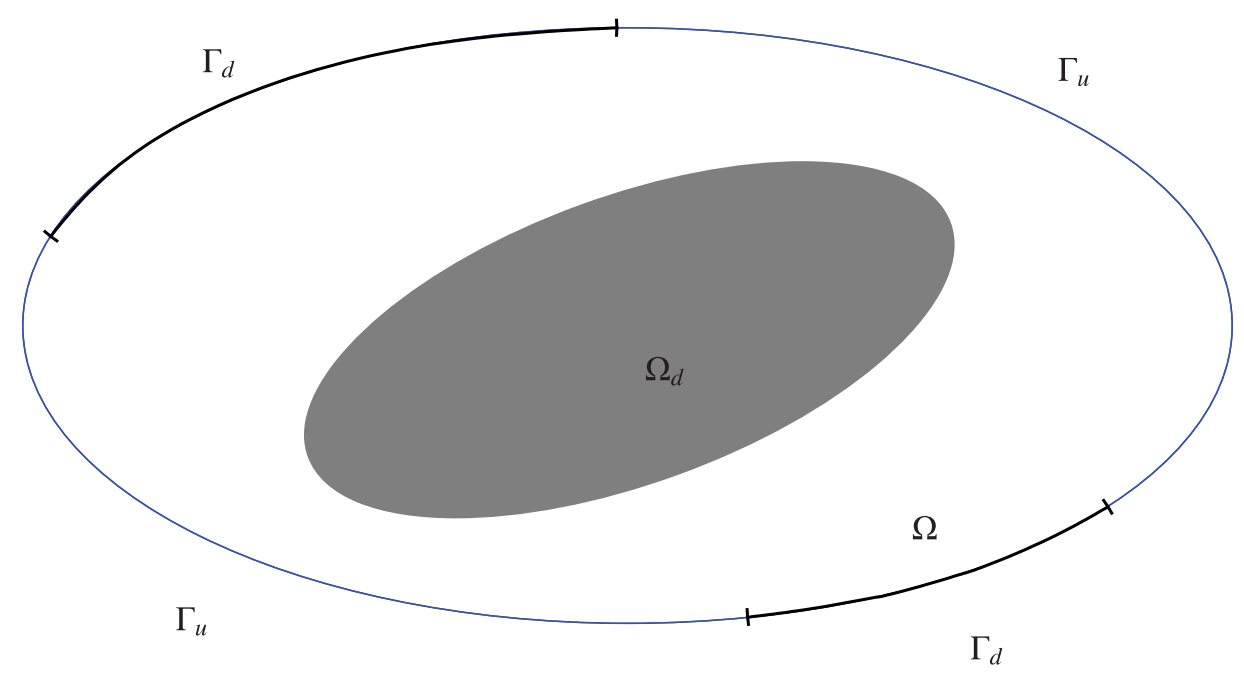

Figure 1. Domain.

$$
\operatorname{div}(\sigma(M))=\underline{0}, \quad \forall M \in \Omega
$$

where $\sigma(M)$ denotes the Cauchy stress tensor.

This tensor is related to the strain tensor $\varepsilon$ through the constitutive law (Hooke's law), namely:

$$
\sigma(M)=\frac{E}{(1+v)} \varepsilon(M)+\frac{v E}{(1+v)(1-2 v)} \operatorname{tr}(\varepsilon(M)) \mathbf{I}
$$

where $E$ is the Young's modulus and $v$ the Poisson's ratio.

The strain tensor $\varepsilon(M)$ is related to the displacement gradient tensor $\nabla \underline{u}$ by the kinematic relations:

$$
\varepsilon(M)=\frac{1}{2}\left(\nabla \underline{u}(M)+\nabla^{T} \underline{u}(M)\right), \quad \forall M \in \Omega
$$

By substituting relations (2) and (3) into the governing Equations (1), the Lamés equations are obtained:

$$
\begin{aligned}
& \mathbf{L}(\underline{u}(M))=\frac{E}{2(1+v)} \underline{\Delta} \underline{u}(M)+\frac{E}{2(1+v)(1-2 v)} \underline{\nabla}(\operatorname{div} \underline{u}(M))=0, \\
& \quad \forall M \in \Omega
\end{aligned}
$$

Let $\underline{n}(M)$ be the outward unit normal vector at a point $M \in \Gamma$ and $\underline{T}(M)=$ $\sigma(M) \cdot \underline{n}(M)$ be the stress vector at the point $M \in \Gamma$.

Displacements are supposed to be measured in $\Omega_{d}$ and the stress vector is supposed to be either null or imposed on $\Gamma_{d}$. This leads to the mathematical formulation of a problem consisting of Equation (4) and the known displacements data and boundary conditions: 


$$
\begin{aligned}
& \underline{u}(M)^{\underline{u}} \underline{u}_{d(M)}, \quad M \in \Omega_{d} \\
& \underline{T}(M)=\underline{T}_{d(M)}, \quad M \in \Gamma_{d}
\end{aligned}
$$

where $\underline{u}_{d}$ and $\underline{T}_{d}$ are prescribed vector functions.

In the above formulation (1), the displacement field is known in $\Omega_{d}$ and the stress vector is known on $\Gamma_{d}$. As a consequence, the boundary $\Gamma_{u}$ is underspecified since both the displacement $\underline{u}$ and the stress vector $\underline{T}$ are unknown.

In the following, we will assume that the boundary conditions on $\Gamma_{d}$ lead to reliable stress vector quantities while the displacement field, evaluated for example by DIC, in $\Omega_{d}$ is considered unreliable due to noisy images or DIC errors.

The Lamés Equation (4) and the prescribed boundary conditions (5) lead to the formulation of the inverse problem in linear isotropic elasticity:

$$
\left\{\begin{array}{lll}
\mathbf{L}(\underline{u}(M)) & =0 & \forall M \in \Omega \\
\underline{u}(M) & =\underline{u}_{d(M)} & \forall M \in \Omega_{d} \\
\underline{T}(M) & =\underline{T}_{d(M)} & \forall M \in \Gamma_{d}
\end{array}\right.
$$

This problem is difficult to solve since it is ill-posed. When it admits a solution, this solution is unique, but it is unfortunately sensitive to small perturbations of the data. Therefore, a regularization method is required in order to solve accurately problem (6).

\section{Fading regularization method}

\subsection{Notations and equivalent formulation of the inverse problem}

Let $H^{1}(\Omega)$ be the Sobolev space of real valued functions in $\Omega$ endowed with the usual Sobolev norm. The restrictions of the functions belonging to $\mathrm{H}^{1}(\Omega)$ to the subset $\Omega_{d} \subset \Omega$ define the space $\mathrm{H}^{1}\left(\Omega_{d}\right)$. The space of traces functions from $\mathrm{H}^{1}(\Omega)$ to $\Gamma$ is denoted by $\mathrm{H}^{1 / 2}(\Gamma)$, while the restrictions of the functions belonging to $\mathrm{H}^{1 / 2}(\Gamma)$ to the subset $\Gamma_{d} \subset \Gamma$ define the space $\mathrm{H}^{1 / 2}\left(\Gamma_{d}\right)$.

Herein, we use the following notation $\mathbf{H}^{1}(\Omega):=\mathrm{H}^{1}(\Omega) \times \mathrm{H}^{1}(\Omega)$, as well as similar notations for the other function spaces employed, i.e. $\mathbf{H}^{1}\left(\Omega_{d}\right):=\mathrm{H}^{1}\left(\Omega_{d}\right) \times \mathrm{H}^{1}\left(\Omega_{d}\right), \quad \mathbf{H}^{1 / 2}(\Gamma):=\mathrm{H}^{1 / 2}(\Gamma) \times \mathrm{H}^{1 / 2}(\Gamma) \quad$ and $\mathbf{H}^{1 / 2}\left(\Gamma_{d}\right):=\mathrm{H}^{1 / 2}\left(\Gamma_{d}\right) \times \mathrm{H}^{1 / 2}\left(\Gamma_{d}\right)$. Finally, we denote by $\mathbf{H}^{-1 / 2}(\Gamma)$ the dual space of $\mathbf{H}^{1 / 2}(\Gamma)$ and by $\mathbf{H}^{-1 / 2}\left(\Gamma_{d}\right)$ the dual space of $\mathbf{H}^{1 / 2}\left(\Gamma_{d}\right)$.

We define the space of solutions of the Lamé's Equation (4) by 


$$
\mathbf{H}(\Omega)=\left\{\underline{u} \in \mathbf{H}^{1}(\Omega) \mid \mathbf{L}(\underline{u}(M))=\underline{0}, M \in \Omega, \text { in a weak sense }\right\}
$$

$\mathbf{H}(\Omega)$ is a closed subspace of $\mathbf{H}^{1}(\Omega)$. We define the space of restrictions in $\Omega_{d}$ of the space of solutions of the Lamés equations $\mathbf{H}(\Omega)$, denoted by $\mathbf{H}\left(\Omega_{d}\right)$, which is endowed with the usual $\mathrm{H}^{1}(\Omega)$ scalar product and its induced norm $\|\cdot\|_{\mathbf{H}\left(\Omega_{d}\right)}$.

We define $\left.\underline{u}\right|_{\Gamma}$ to be the traces of elements $\underline{u} \in \mathbf{H}(\Omega)$ on $\Gamma$ and their associated stress vector $\underline{T}(\underline{u})$ on $\Gamma$ where $\underline{T}(\underline{u}):=\sigma(\underline{u}) \cdot \underline{n}$.

Next, we generate the space of solution functions:

$$
\begin{aligned}
\mathbf{H}(\Gamma) & =\left\{\underline{U}=(\underline{v}, \underline{u}, \underline{T}(\underline{u})) \in \mathbf{H}^{1}(\Omega) \times \mathbf{H}^{1 / 2}(\Gamma) \times \mathbf{H}^{-1 / 2}(\Gamma)|\underline{v} \in \mathbf{H}(\Omega), \underline{v}|_{\Gamma}\right. \\
& \left.=\underline{u},\left.\underline{T}(\underline{v})\right|_{\Gamma}=\underline{T}(\underline{u})\right\}
\end{aligned}
$$

and it can be shown that the space of solution functions $\mathbf{H}(\Gamma)$ is a closed subspace of $\mathbf{H}^{1}(\Omega) \times \mathbf{H}^{1 / 2}(\Gamma) \times \mathbf{H}^{-1 / 2}(\Gamma)$ with respect to the scalar product $\left\langle\mathbf{U}, \mathbf{U}^{\prime}\right\rangle_{\mathbf{H}(\Gamma)}$ (Equation (9)) and its induced norm $\|\cdot\|_{\mathbf{H}(\Gamma)}$.

$$
\begin{aligned}
\left\langle\underline{U}, \underline{U^{\prime}}\right\rangle_{\mathbf{H}(\Gamma)}= & \frac{1}{\operatorname{meas}(\Omega)}<\underline{v}, \underline{v^{\prime}}>_{H^{1}(\Omega)}+\frac{1}{\operatorname{meas}(\Gamma)}<\underline{u}, \underline{u}^{\prime}>_{H^{1 / 2}(\Gamma)} \\
& +\frac{l_{0}^{2}}{\operatorname{meas}(\Gamma)} \frac{1}{E^{2}}<\underline{T}, \underline{T^{\prime}}>_{H^{-1 / 2}(\Gamma)}, \\
& \forall\left(\underline{U}=(\underline{v}, \underline{u}, \underline{T}), \underline{U^{\prime}}=\left(\underline{v}^{\prime}, \underline{u^{\prime}}, \underline{T^{\prime}}\right)\right) \in \mathbf{H}(\Gamma) \times \mathbf{H}(\Gamma)
\end{aligned}
$$

where $l_{0}$ is a characteristic length of the considered domain $\Omega$.

An equivalent formulation of the inverse problem (6) reads as

$$
\text { Find } \underline{U}=(\underline{v}, \underline{u}, \underline{T}) \in \mathbf{H}(\Gamma) \text { such that }\left.\underline{v}\right|_{\Omega_{d}}=\underline{u}_{d} \text { and }\left.\underline{T}\right|_{\Gamma_{d}}=\underline{T}_{d} \text {. }
$$

An idea for solving problem (6) consists of seeking the best solution satisfying the measurement data (5a) on the accessible domain $\Omega_{d}$, provided that the equilibrium Equation (4) and the boundary conditions (5b) on the boundary part $\Gamma_{d}$ are fulfilled. This leads to defining the solution of the inverse problem (4), (5a) and (5b) in terms of an approximate solution which solves the following optimization problem:

$$
\mid \begin{aligned}
& \operatorname{Given}\left(\underline{u}_{d}, \underline{T}_{d}\right) \in \mathbf{H}\left(\Omega_{d}\right) \times \mathbf{H}^{-\frac{1}{2}}\left(\Gamma_{d}\right), \text { find } \underline{U}=(\underline{v}, \underline{u}, \underline{T}) \in \mathbf{H}(\Gamma) \text { such that } \\
& \quad J(\underline{U}) \leq J(\underline{V}), \forall \underline{V}=\left(\underline{v^{\prime}}, \underline{u^{\prime}}, \underline{T^{\prime}}\right) \in \mathbf{H}(\Gamma) \\
& \text { under the equality constraint }\left.\underline{T}\right|_{\Gamma_{d}}=\underline{T}_{d}, \\
& \text { where } J(\cdot): \mathbf{H}(\Gamma) \rightarrow\left[0, \infty\left[, \quad J(\underline{V})=\left\|\left.\underline{v}^{\prime}\right|_{\Omega_{d}}-\underline{u}_{d}\right\|_{\mathbf{H}\left(\Omega_{d}\right)}^{2} .\right.\right.
\end{aligned}
$$




\subsection{Continuous formulation of the fading regularization algorithm}

Problem (11) remains also ill-posed even if it admits a unique solution. Therefore, an iterative regularizing method is introduced to solve it. The method chosen is a generalization of the inverse technique introduced by Cimetière et al. (Cimetière et al., 2001, 2000) to solve the Cauchy problem for the Laplace equation. It can be considered as an iterative Tikhonovtype regularization method.

Given $c>0$ and $\underline{U}^{0} \in \mathbf{H}(\Gamma)$ the iterative algorithm reads as:

$$
\mid \begin{aligned}
& \text { Find } \underline{U}^{k+1}=\left(\underline{v}^{k+1}, \underline{u}^{k+1}, \underline{T}^{k+1}\right) \in \mathbf{H}(\Gamma) \text { such that : } \\
& J_{c}^{k}\left(\underline{U}^{k+1}\right) \leq J_{c}^{k}(\underline{V}) \quad \forall \underline{V}=\left(\underline{v^{\prime}}, \underline{u^{\prime}}, \underline{T^{\prime}}\right) \in \mathbf{H}(\Gamma), \text { with } \\
& J_{c}^{k}(\underline{V})=\left\|\left.\underline{v}^{\prime}\right|_{\Omega_{d}}-\underline{u}_{d}\right\|_{\mathbf{H}\left(\Omega_{d}\right)}^{2}+c\left\|\underline{V}-\underline{U}^{k}\right\|_{\mathbf{H}(\Gamma)}^{2}, \\
& \text { under the equality constraint } \underline{T^{\prime}}=\underline{T}_{d} \text { on } \Gamma_{d} .
\end{aligned}
$$

In this iterative process, the Lamé's Equation (4) is taken into account exactly since at each step the search for the optimal element is performed in space $\mathbf{H}(\Gamma)$. The boundary conditions concerning the stress vectors $\underline{T}$ on $\Gamma_{d}$ are also taken into account exactly due to the equality constraint. The functional in (12) is composed of two terms which play different roles. The first one acts only in $\Omega_{d}$ and represents the gap between the optimal displacement field $\underline{v}^{k+1}$ and the given data. It relaxes the data which can be possibly perturbed by measurement noises (relaxation term). The second one acts in the whole domain $\Omega$ and on the boundary $\Gamma$ and not only where the information are to be completed. This term is a regularization term, that controls the distance between the new optimal solution and the previous optimal one, and tends to zero as iterations go on.

So, at each step $k+1$, the optimal element $\underline{U}^{k+1}$ obtained is an exact solution of the Lamés Equation (4), which satisfies the boundary conditions (5b) on $\Gamma_{d}$ and is close to the data $\underline{u}^{d}$ in $\Omega_{d}$.

\subsection{Discrete formulation of the fading regularization algorithm using the MFS}

Solving (11) for any geometry and boundary conditions requires to discretize the space $H(\Gamma)$ of solutions. In this paper, it is accomplished in finite dimension with a meshless method, namely the MFS.

The main idea of the MFS consists of approximating the unknown displacement vector on $\bar{\Omega}=\Omega \cup \Gamma$ by a linear combination of fundamental solutions of the Lamé's Equation (4). In the two-dimensional case, a fundamental solution is given by Berger and Karageorghis (Berger \& 
Karageorghis, 2001) at a point $M$, inside the domain, as a function of a singular point $Q$, outside the domain, i.e.:

$$
\underline{u}^{j}(P, Q)=u_{i j}(P, Q) \underline{e}_{i}, \quad P \in \bar{\Omega}, \quad Q \in \mathbb{R}^{2} \backslash \bar{\Omega}, \quad j=1,2
$$

with

$$
\begin{aligned}
& u_{i j}(P, Q)=-\frac{1}{8 \pi G(1-\bar{v})}\left((3-4 \bar{v}) \ln r(P, Q) \delta_{i j}-\frac{\left(x_{i_{P}}-x_{i_{Q}}\right)\left(x_{j_{P}}-x_{j_{Q}}\right)}{r^{2}(P, Q)}\right), \\
& \quad i, j=1,2
\end{aligned}
$$

where $\underline{e}_{i}, i=1,2$, is the unit vector along the $x_{i}$-axis and $\delta_{i j}$ is the Kronecker delta tensor. $r(P, Q)=\sqrt{\left(x_{1_{P}}-x_{1_{Q}}\right)^{2}+\left(x_{2_{P}}-x_{2_{Q}}\right)^{2}}$ represents the Euclidean distance between the point $\mathrm{P}$ and the source point $\mathrm{Q}, G=$ $\frac{E}{2(1+v)}$ is the shear modulus and $\bar{v}$ is introduced to distinguish the plane strain state $(\bar{v}=v)$ and the plane stress state $\left(\bar{v}=\frac{v}{1+v}\right)$.

With respect to $N$ source points located outside the physical domain, $Q^{l} \in \mathbb{R}^{2} \backslash \bar{\Omega}, 1 \leq l \leq N$, the displacement field at a point $\mathrm{M}$ is approximated by:

$$
\underline{u}(P) \approx \underline{u}(\underline{a}, \underline{b}, \underline{Q} ; P)=\sum_{l=1}^{N} a_{l} \underline{u}^{1}\left(P, Q^{l}\right)+b_{l} \underline{u}^{2}\left(P, Q^{l}\right), \quad M \in \bar{\Omega}
$$

where $\underline{a}=\left(a_{1}, \ldots, a_{N}\right), \underline{b}=\left(b_{1}, \ldots, b_{N}\right)$ and $\underline{Q}$ is the $2 N$-vector containing the coordinates of the source points $Q^{l}, 1 \leq l \leq N$.

On taking into account the kinematic relations (3), the constitutive Equation (2), the definitions of the components of the stress vector $\underline{T}(M)$, and the fundamental solution (13), then traction vector is approximated on $\Gamma$ by:

$$
\underline{T}(P) \approx \underline{T}(\underline{a}, \underline{b}, \underline{Q} ; P)=\sum_{l=1}^{N} a_{l} \underline{T}^{1}\left(P, Q^{l}\right)+b_{l} \underline{T}^{2}\left(P, Q^{l}\right), \quad P \in \Gamma
$$

where

$$
\underline{T}^{\mathrm{j}}(P, Q)=T_{i j}(P, Q) \underline{e}_{i}, \quad P \in \Gamma, \quad Q \in \mathbb{R}^{2} \backslash \bar{\Omega}, \quad j=1,2
$$

with 


$$
\begin{aligned}
T_{1 j}(P, Q)= & 2 G\left(\frac{1-\bar{v}}{1-2 \bar{v}} \frac{\partial u_{1 j}(P, Q)}{\partial x_{1}}+\frac{\bar{v}}{1-2 \bar{v}} \frac{\partial u_{2 j}(P, Q)}{\partial x_{2}}\right) n_{1}(P) \\
& +G\left(\frac{\partial u_{1 j}(P, Q)}{\partial x_{2}}+\frac{\partial u_{2 j}(P, Q)}{\partial x_{1}}\right) n_{2}(P), \quad j=1,2 \\
T_{2 j}(P, Q)= & G\left(\frac{\partial u_{1 j}(P, Q)}{\partial x_{2}}+\frac{\partial u_{2 j}(P, Q)}{\partial x_{1}}\right) n_{1}(P) \\
& +2 G\left(\frac{\bar{v}}{1-2 \bar{v}} \frac{\partial u_{1 j}(P, Q)}{\partial x_{1}}+\frac{1-\bar{v}}{1-2 \bar{v}} \frac{\partial u_{2 j}(P, Q)}{\partial x_{2}}\right) n_{2}(P), \quad j=1,2 .
\end{aligned}
$$

Further, according to the fading regularization algorithm, at each step $k \geq 0$ of the minimization problem (12), one has to approximate both the known data $\underline{u}_{d}$ in $\Omega_{d}$, the known boundary condition $\underline{T}_{d}$ on $\Gamma_{d}$, the unknown displacement $\underline{u}$ in $\bar{\Omega} \backslash \Omega_{d}$ and the unknown boundary condition $\underline{T}$ on $\Gamma_{u}$, at the same time accounting for the given perturbed data. To do this, we collocate the data at a set of $M_{d}$ points: $M_{\Omega_{d}}$ points are located inside the domain $\left(P^{l} \in \Omega_{d}, 1 \leq l \leq M_{\Omega_{d}}\right)$ and $M_{\Gamma_{d}}$ points are located at the boundary ( $P^{l} \in \Gamma_{d}, 1 \leq l \leq M_{\Gamma_{d}}$ ). We also express the MFS approximations (14) and (15) for the unknown data at a set of $M_{u}$ points: $M_{\Omega \backslash \Omega_{d}}$ points inside the domain where the displacement field is unknown and $M_{\Gamma_{u}}$ points at the boundary where the stress vector is unknown. Consequently, at each step $k \geq 0$, the minimization problem (12) is reduced to a linear minimization problem with respect to the corresponding unknown MFS constants.

Remarks:

i It can be noticed that the use of the MFS numerical method induces regular $C^{\infty}(\Gamma)$ piecewize approximations for the traction vector $T$ and allows us to replace the $H^{-1 / 2}(\Gamma)$ norm by the $L^{2}(\Gamma)$ norm.

ii The equivalence of norms in finite dimension allows us to replace the different norms involved in the functional (12) by their corresponding $L^{2}$ norms.

\section{Numerical results and discussion}

We present the numerical results obtained using the MFS-fading regularization algorithm described in the previous section. First, various relatively simple cases are considered, for which an analytical solution $\underline{u}^{a n}$ is known, allowing us to generate the data (5) on the grid $\Omega_{d}$ and the boundary part $\Gamma_{d}$.

The following control quantities are used to estimate the accuracy of the proposed algorithm and the convergence of the iterative process: 
(i) the relaxation term of the functional (12):

$$
J_{\Omega_{d}}\left(u^{k+1}\right)=\left\|\left.u^{k+1}\right|_{\Omega_{d}}-\underline{u}_{d}\right\|_{H\left(\Omega_{d}\right)}^{2} ;
$$

(ii) the regularization term of the functional (13):

$$
J_{\text {reg. }}^{k}\left(\underline{U}^{k+1}\right)=c\left\|\underline{U}^{k+1}-\underline{U}^{k}\right\|_{H(\Gamma)}^{2} ;
$$

(iii) the value $J^{k}\left(\underline{U}^{k+1}\right)=J_{\Omega_{d}}\left(\underline{U}^{k+1}\right)+J_{\text {reg. }}^{k}\left(\underline{U}^{k+1}\right)$ of the functional (12) for the optimal element $\underline{U}^{k+1}$;

(iv) the $L^{2}$-relative error for $\underline{v}$ in $\Omega$

$$
u_{e r r o r}^{\Omega}=\frac{\left\|\underline{\underline{v}}-\underline{u}^{a n}\right\|_{L^{2}(\Omega)}}{\left\|\underline{u}^{a n}\right\|_{L^{2}(\Omega)}} ;
$$

(v) the $L^{2}$-relative error for $\underline{u}$ on $\Gamma$

$$
u_{\text {error }}^{\Gamma}=\frac{\left\|\underline{u}-\underline{u}^{a n}\right\|_{L^{2}(\Gamma)}}{\left\|\underline{u}^{a n}\right\|_{L^{2}(\Gamma)}} ;
$$

(vi) the $L^{2}$-relative error for $\underline{T}$ on $\Gamma$

$$
T_{\text {error }}=\frac{\left\|\underline{T}-\underline{T}^{a n}\right\|_{L^{2}(\Gamma)}}{\left\|\underline{T}^{a n}\right\|_{L^{2}(\Gamma)}} .
$$

Three properties of the functional terms in the minimizing sequence can be established without the assumption that the data $\underline{u}^{d}$ is compatible, namely

(a) $J_{\Omega_{d}}\left(\underline{U}^{k+1}\right)$ is monotonically decreasing, i.e.

$$
J_{\Omega_{d}}\left(\underline{U}^{k+1}\right) \leq J_{\Omega_{d}}\left(\underline{U}^{k}\right), \quad \forall k \geq 0 .
$$

(b) The second one $J_{\text {reg. }}^{k}\left(\underline{U}^{k+1}\right)$ is monotonically decreasing as soon as $c>0$, i.e.

$$
J_{\text {reg. }}^{k}\left(\underline{U}^{k+1}\right) \leq J_{\text {reg. }}^{k-1}\left(\underline{U}^{k}\right), \quad \forall k \geq 0 .
$$

(c) The sequence defined by the values of the functional $J$ for each optimal element $\left(\underline{U}^{k+1}\right)$ is also monotonically decreasing as soon as $c>0$, i.e.

$$
J^{k}\left(\underline{U}^{k+1}\right) \leq J^{k-1}\left(\underline{U}^{k}\right), \quad \forall k \geq 0 .
$$

\subsection{Unconfined uniaxial compression test of a rectangular specimen}

As a first example, an unconfined compression test is selected (Figure 2). The analytical solution for the displacement field $\underline{u}^{a n}=\left(u_{1}^{a n}, u_{2}^{a n}\right)$ is the following: 


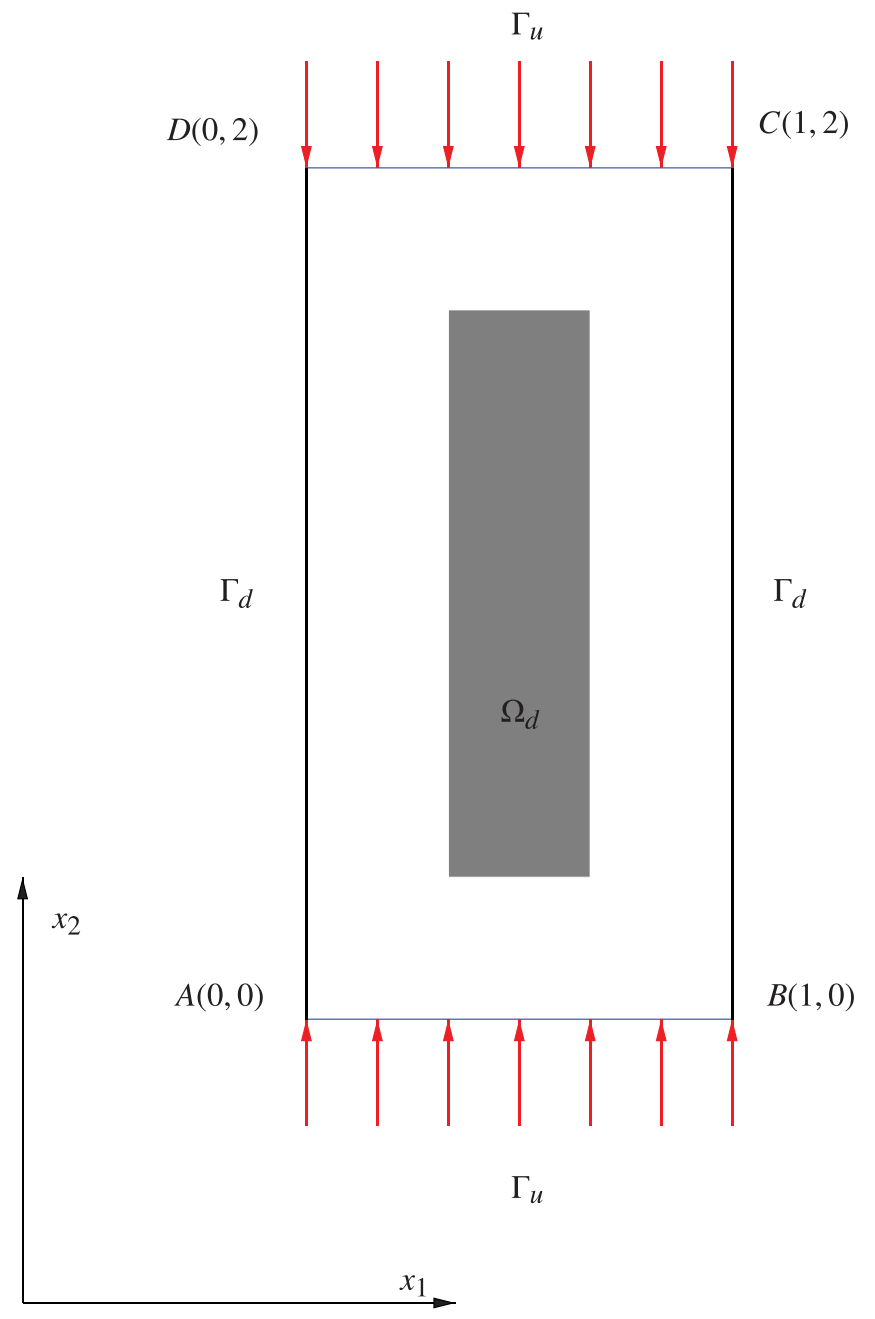

Figure 2. Compressed pavement.

$$
\begin{aligned}
& u_{1}^{a n}\left(x_{1}, x_{2}\right)=\frac{v \sigma_{0}}{E} x_{1}, \quad\left(x_{1}, x_{2}\right) \in \bar{\Omega}, \\
& u_{2}^{a n}\left(x_{1}, x_{2}\right)=-\frac{\sigma_{0}}{E} x_{2}, \quad\left(x_{1}, x_{2}\right) \in \bar{\Omega}
\end{aligned}
$$

in the domain $\bar{\Omega}=\left\{\underline{x}=\left(x_{1}, x_{2}\right) \mid 0 \leq x_{1} \leq 1\right.$ and $\left.0 \leq x_{2} \leq 2\right\}$ with $E=1$ $\mathrm{N} . \mathrm{m}^{-2}, v=0.2$, and $\sigma_{0}=0.1 \mathrm{~N} . \mathrm{m}^{-2}$.

The data grid $\Omega_{d}$ is defined by parameter $\zeta$ such that meas $\left(\Omega_{d}\right)=$ $\zeta$ meas $(\Omega)$ and $\Gamma$ is the boundary defined by $\Gamma=\Gamma_{d} \cup \Gamma_{u}$ with $\Gamma_{d}=\{\underline{x} \in$ $\Gamma \mid x_{1}=0$ and $\left.0 \leq x_{2} \leq 2\right\} \cup\left\{\underline{x} \in \Gamma \mid x_{1}=1\right.$ and $\left.0 \leq x_{2} \leq 2\right\}$ and $\Gamma_{u}=$ $\left\{\underline{x} \in \Gamma \mid 0 \leq x_{1} \leq 1\right.$ and $\left.x_{2}=0\right\} \cup\left\{\underline{x} \in \Gamma \mid 0 \leq x_{1} \leq 1\right.$ and $\left.x_{2}=2\right\}$.

The corresponding MFS parameters have been set as $N=90$ on $\tilde{\Gamma}=$ $\left\{\underline{x}=\left(x_{1}, x_{2}\right) \in \mathbb{R}^{2} \mid x_{1}^{2}+x_{2}^{2}=d^{2}\right\} \quad$ and $\quad d=10, \quad$ whilst $\quad M_{d}=100$ and $M_{u}=50$. 


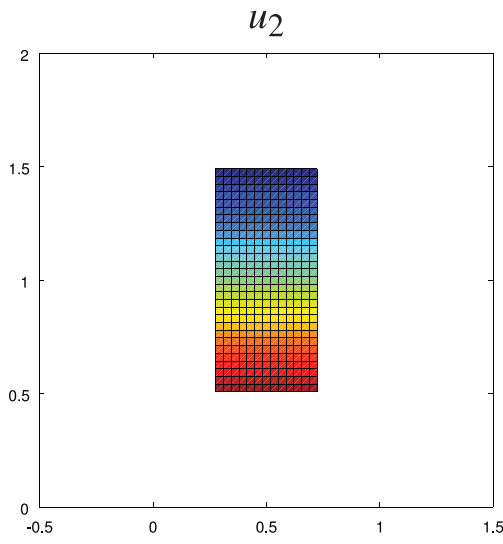

(a)

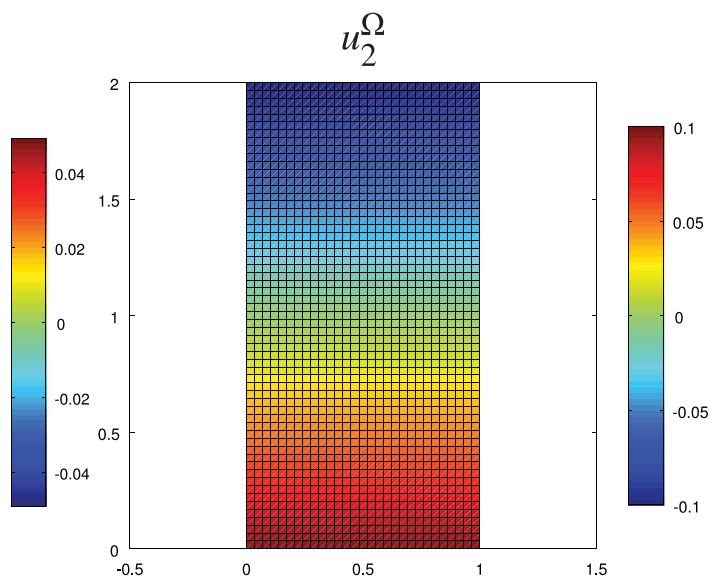

(b)

Figure 3. Reconstruction of the vertical component of the displacement, (a) $u_{2}$ in $\Omega_{d}$, and (b) $u_{2}^{\Omega}$ in $\Omega$, for $c=10^{-3}$.

If it is not specified, the data grid $\Omega_{d}$ is defined by the parameter $\zeta=\frac{1}{4}$, which means that $\Omega_{d}$ is four-times smaller than $\Omega$. The domain $\Omega$ is discretized with $(30 \times 60)$ points.

\subsubsection{Exact data}

In the present case, the data in $\Omega_{d}$ are generated from the analytical solution for the displacement field $\underline{u}^{a n}$ (24) and the boundary conditions for its associated stress vector on $\Gamma_{d}$ are generated from $\underline{T}^{a n}$ and both are not blurred. As expected, the proposed algorithm allows one to reconstruct the displacement field on the data grid $\Omega_{d}$ and retrieve the displacements in the entire domain $\Omega$. Figure $3(\mathrm{a}, \mathrm{b})$ shows the reconstructions of the vertical component of the displacement $u_{2}$ in $\Omega_{d}\left(u_{2}\right)$ and in $\Omega\left(u_{2}^{\Omega}\right)$, for $c=10^{-1}$ and 67 iterations, respectively.

Figure $4(\mathrm{a}, \mathrm{b})$ shows the perfect reconstructions of the vertical component of displacement $u_{2}$ and the vertical component of the stress vector $T_{2}$ on $\Gamma$, respectively. ${ }^{1}$

In these figures the results are displayed on the whole boundary $\Gamma$ thanks to the use of a curvilinear abscissa $s$, growing, respectively, from 0 to 1 as $M$ describes $\widehat{A B}$, from 1 to 3 as $M$ describes $\widehat{B C}$, from 3 to 4 as $M$ describes $\widehat{C D}$, and from 4 to 6 as $M$ describes $\widehat{D A}$ (Figure 2).

4.1.1.1 Stopping criterion. For both exact and noisy data, a stopping criterion is needed to end the iterative process before the noise enters significantly the numerical solution and hence the latter becomes oscillatory/unstable. 


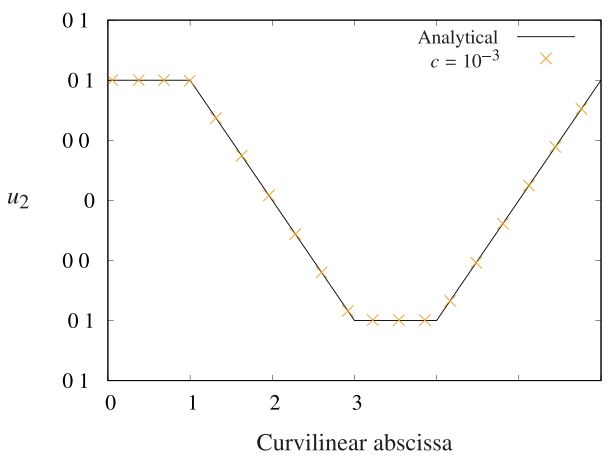

(a)

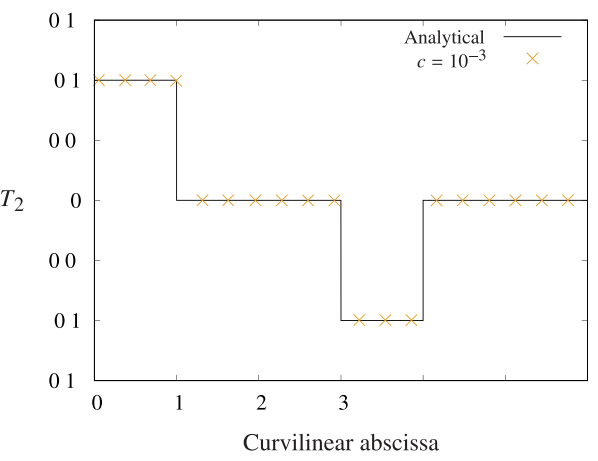

(b)

Figure 4. (a) Analytical, $u_{2}^{a n}$, and numerical vertical components of displacement, $u_{2}$, and (b) analytical, $T_{2}^{a n}$, and numerical vertical components of stress vector, $T_{2}$, retrieved on the boundary $\Gamma$ for $c=10^{-3}$.

Figure 5 represents the evolution of the control quantities $J_{\Omega_{d}}\left(\underline{U}^{k+1}\right)$, $J_{\text {reg. }}\left(\underline{U}^{k+1}\right)$ and $J\left(\underline{U}^{k+1}\right)$ versus the number of iterations $k$ for the parameter $c=10^{-1}$. We note that for $k \geq 67$ the quantities $J_{\Omega_{d}}\left(\underline{U}^{k+1}\right), J_{\text {reg. }}\left(\underline{U}^{k+1}\right)$ and $J\left(\underline{U}^{k+1}\right)$, as well as the errors $\underline{u}_{\text {error }}^{\Omega}, \underline{u}_{\text {error }}^{\Gamma}$ and $\underline{T}_{\text {error }}$, remain constant. We also observe that the method converges.

The iteration at which the iterative process is stopped, is determined by using the quantity $J_{\text {reg. }}\left(\underline{U}^{k+1}\right)$, where $\underline{U}^{k+1}$ is the optimal element obtained

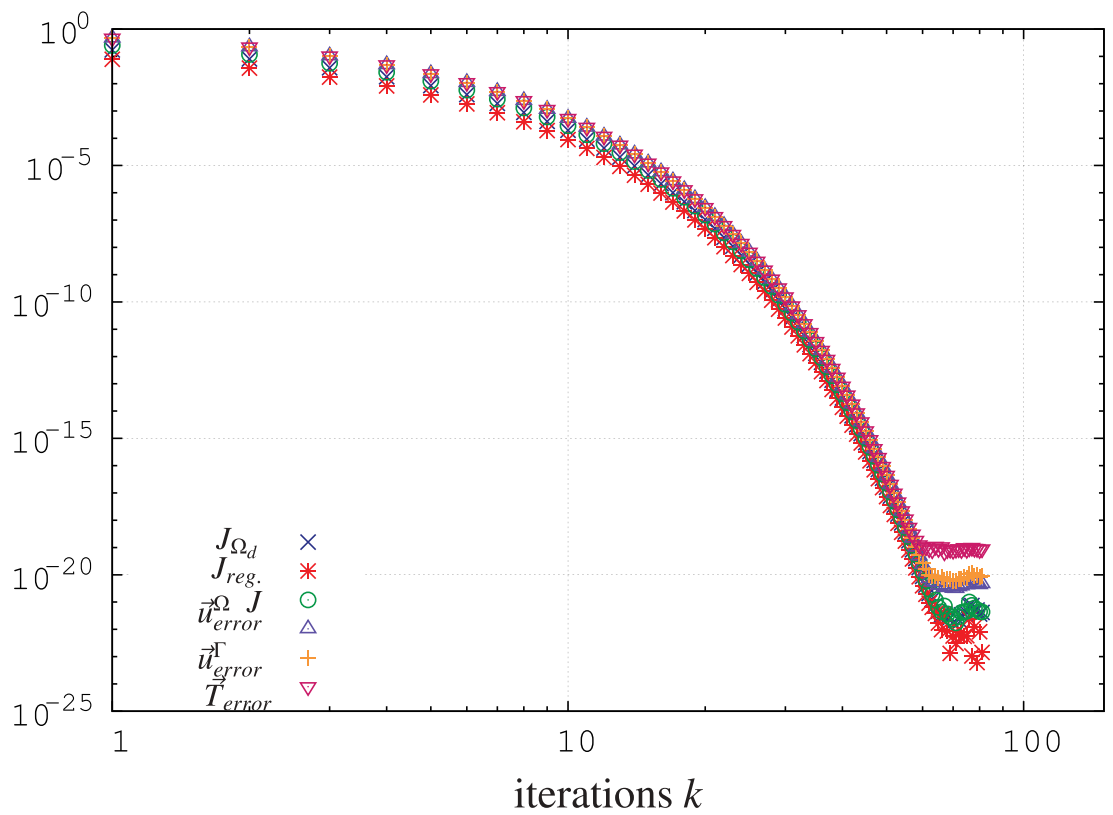

Figure 5. Evolution of the control terms with respect to the number of iterations, $k$, for $c=10^{-1}$. 
at the $(k+1)^{\text {th }}$ step. As expected, the control quantity $J_{\text {reg. }}$ decreases when $k<67$ and then becomes constant with only a few oscillations caused by numerical approximations, for $k>67$. We search the step $k_{\text {opt }}$ for which the regularization term $J_{\text {reg. }}$ increases, i.e $J_{\text {reg. }}\left(\underline{U}^{k+1}\right)>J_{\text {reg. }}\left(\underline{U}^{k}\right)$ and hence the iterative process is stopped at $k=k_{o p t}=67$. It must be emphasized that this stopping criterion is blind because, when calculating the quantity $J_{\text {reg. }}$, we do not need to know the analytical solution. Indeed, it is sufficient to know two successive optimal elements and compare them.

We observe that oscillations occur in the term $J_{\text {reg. }}$. because of numerical approximations; however, this term becomes negligible compared to $J_{\Omega_{d}}$ and tends to zero. This proves that the algorithm converges actually. The residual error $J_{\Omega_{d}}$ (relaxation term) and the term $J$ decrease during the iterative process. After convergence is achieved, the relaxation term $J_{\Omega_{d}}$ remains constant and this corresponds to a numerical approximation error.

4.1.1.2 Influence of parameter $c$. It is also necessary to investigate the influence of parameter $c$, which defines the relative weight of the regularization term $J_{\text {reg. }}$ compared to the relaxation term $J_{\Omega_{d}}$. Figure $6(\mathrm{a}, \mathrm{b})$ shows the reconstructions of the vertical component of displacement $u_{2}$ and the vertical component of the stress vector $T_{2}$ on $\Gamma$, for various values of $c$, respectively.

It can be seen from Table 1, that the value of parameter $c$ affects the number of iterations $k$ required to obtain convergence. Table 1 lists the results, obtained for a wide range of regularization parameter $c$, by specifying the number of iterations necessary to achieve convergence and the corresponding errors $u_{\text {error }}^{\Omega}, u_{\text {error }}^{\Gamma}$ and $T_{\text {error }}$. We note that the errors in $\underline{u}$

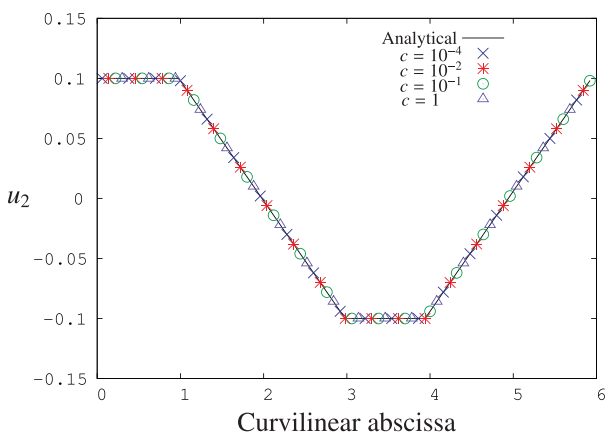

(a)

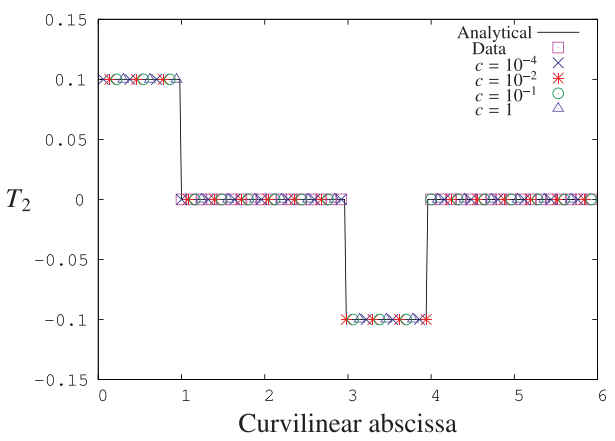

(b)

Figure 6. (a) Analytical, $u_{2}^{a n}$, and numerical vertical components of displacement, $u_{2}$, and (b) analytical, $T_{2}^{a n}$, and numerical vertical components of the stress vector, $T_{2}$, retrieved on the boundary $\Gamma$ for various values of $c$. 
Table 1. Influence of the regularization parameter $c$ on the number of iterations, $k$, required to achieve convergence, and on the errors $u_{\text {error }}^{\Omega}, u_{\text {error }}^{\Gamma}$ and $T_{\text {error }}$.

\begin{tabular}{ccccc}
$c$ & $k$ & $u_{\text {error }}^{\Omega}$ & $u_{\text {error }}^{\Gamma}$ & $T_{\text {error }}$ \\
\hline $10^{-10}$ & 2 & $2.07 \times 10^{-21}$ & $3.68 \times 10^{-21}$ & $3.40 \times 10^{-20}$ \\
$10^{-8}$ & 4 & $9.93 \times 10^{-21}$ & $1.69 \times 10^{-20}$ & $4.24 \times 10^{-20}$ \\
$10^{-6}$ & 6 & $2.32 \times 10^{-21}$ & $4.02 \times 10^{-21}$ & $9.24 \times 10^{-21}$ \\
$10^{-4}$ & 7 & $2.84 \times 10^{-21}$ & $7.55 \times 10^{-21}$ & $2.40 \times 10^{-20}$ \\
$10^{-3}$ & 11 & $5.31 \times 10^{-20}$ & $8.86 \times 10^{-20}$ & $1.53 \times 10^{-19}$ \\
$10^{-2}$ & 18 & $2.65 \times 10^{-20}$ & $4.66 \times 10^{-20}$ & $1.22 \times 10^{-19}$ \\
$10^{-1}$ & 67 & $3.41 \times 10^{-21}$ & $6.34 \times 10^{-21}$ & $6.88 \times 10^{-20}$ \\
\hline
\end{tabular}

and $T$ are identical for each value of the parameter $c$ listed in Table 1. This confirms that the algorithm converges to the same solution, regardless of the value of $c$.

Figure 7 shows the evolution of the control quantities introduced in Equations (16) and (17) versus $\frac{k}{c}$, for three values of $c$. We notice that, for each $c$, these quantities become negligible as the number of iterations required to achieve convergence is reached.

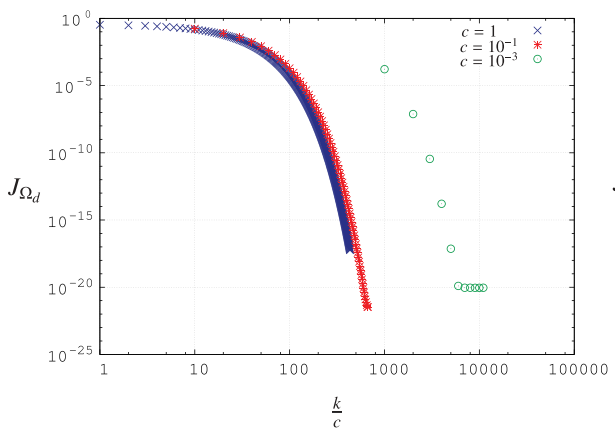

(a)

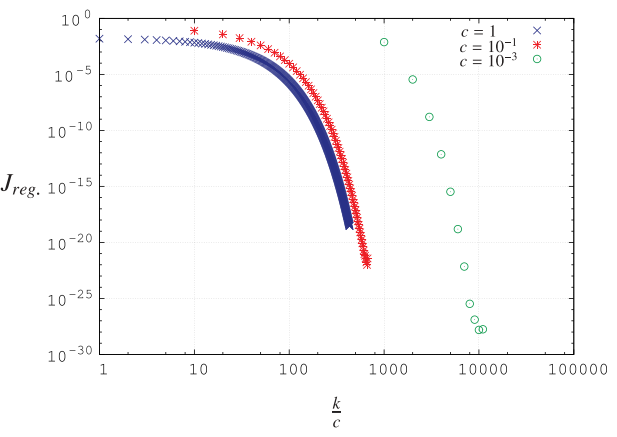

(b)

Figure 7. Evolution of the norms (a) $J_{\Omega_{d}}$, and (b) $J_{\text {reg., }}$ as functions of $\frac{k}{c}$, for various values of $c$.

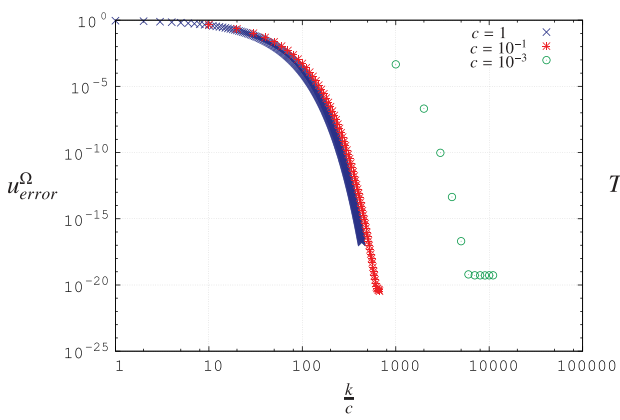

(a)

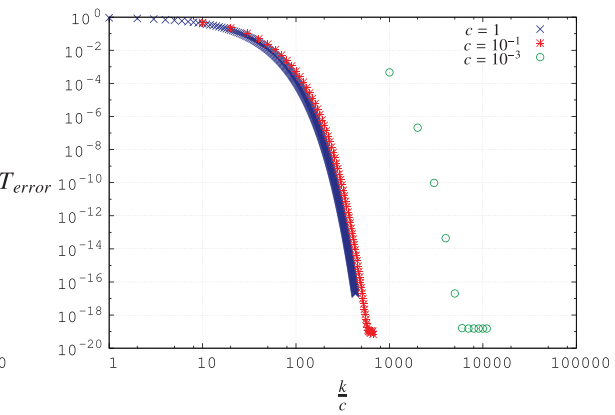

(b)

Figure 8. Evolution of the errors (a) $u_{\text {error }}^{\Omega}$ and (b) $T_{\text {error, }}$ as functions of $\frac{k}{c}$, for various values of c. 
Analogously and for comparison purposes, Figure 8 shows the evolution of the relative errors introduced by Equations (18) and (20), as functions of $\frac{k}{c}$ for various values of $c$. Each quantity reaches very small values, see Figure $8(\mathrm{a}, \mathrm{b})$. This again confirms that the algorithm converges to the same solution, regardless of the value of $c$, which acts only on the convergence rate.

\subsubsection{Reconstruction from a displacement field disturbed by a rigid body motion}

During real experiments, the displacement field data may be disturbed by a rigid body motion. It is thus necessary to investigate how the reconstructions are influenced by this kind of perturbations.

A rigid body motion $\underline{u}_{r i g}$ is the composition of a translation and a rotation. Characterizing the rigid body translation by its two components $a$ and $b$ and the rigid body rotation by the angle $\alpha$, the components $u_{1}^{\text {rig }}$ and $u_{2}^{\text {rig }}$ of the displacement can be written as follows:

$$
\begin{aligned}
& u_{1}^{r i g}=a+x_{M} \cos \alpha-y_{M} \sin \alpha-x_{M} \\
& u_{2}^{r i g}=b+x_{M} \sin \alpha+y_{M} \cos \alpha-y_{M}
\end{aligned}
$$

where $\left(x_{M}, y_{M}\right)$ are the coordinates of a point $M$ in $\Omega$ and the origin $O$ is considered as the centre of rotation.

Figure $9(\mathrm{a}, \mathrm{b})$ present the deformable part of the displacement components $u_{1}^{\text {def }} \Omega$ and $u_{2}^{\text {def }} \Omega$ obtained by the MFS-FRM algorithm in $\Omega$, when data $u^{d}$ in $\Omega_{d}$ are disturbed by the rigid body motion defined by (25), where $a=0.02, b=0.05$ and $\alpha=0.1^{\circ}$.

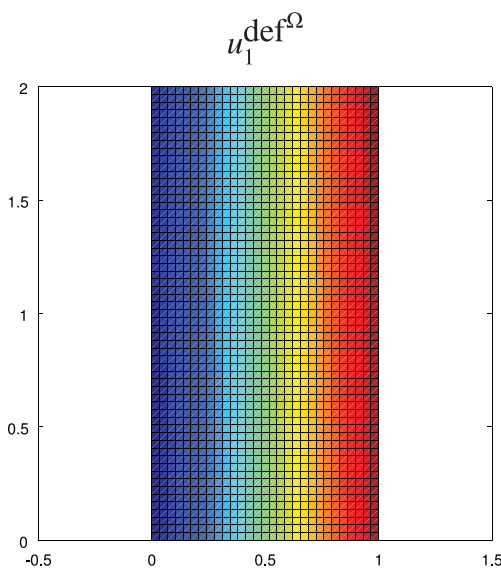

(a)

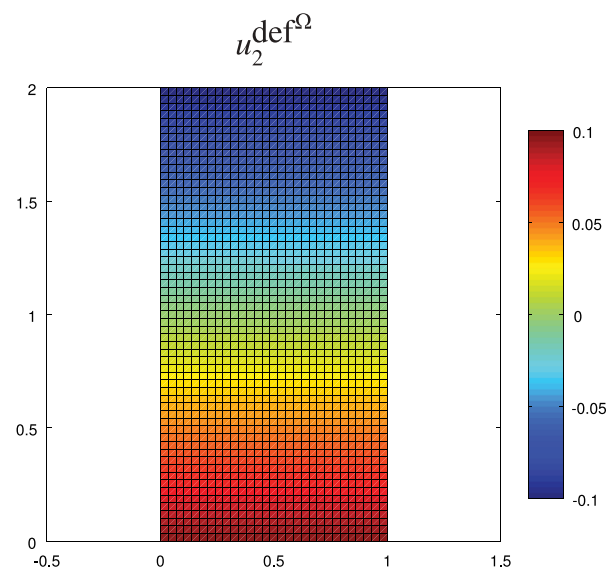

(b)

Figure 9. (a) Deformable part of the horizontal displacement, $u_{1}^{\text {def }^{\Omega}}$, and (b) deformable part of the vertical displacement, $u_{2}^{\text {def }}$, in $\Omega$, for $c=10^{-1}$. 


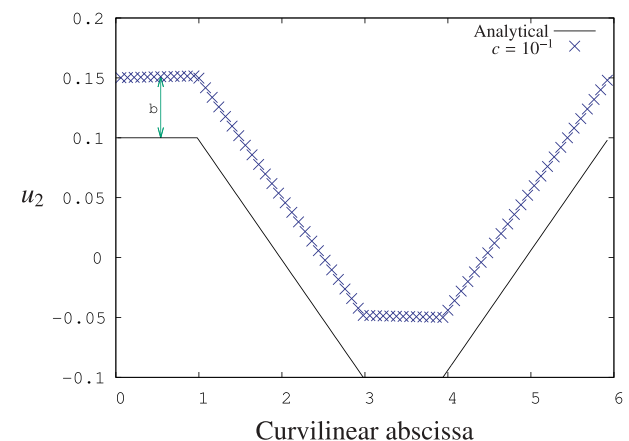

(a)

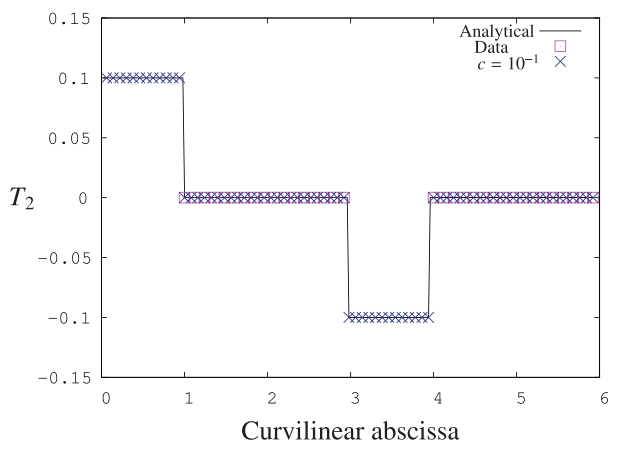

(b)

Figure 10. (a) Analytical, $u_{2}^{a n}$, and numerical, $u_{2}$, vertical displacements and (b) analytical, $T_{2}^{a n}$, and numerical, $T_{2}$, stress vector vertical components, retrieved on the boundary $\Gamma$, when the data are perturbed by a rigid body motion, for $c=10^{-1}$.

The vertical components of the boundary conditions $u_{2}$ and $T_{2}$ on $\Gamma$, obtained by the MFS-FRM algorithm, are represented on the Figure 10(a,b), respectively.

We remark that the reconstructed displacements always contain the rigid body motion, however, as expected, stress vectors are not influenced and correspond to the exact solution. With a post-processing, using a nonlinear optimization procedure, we determine the parameters $a=0.0201$, $b=0.0499$ and $\alpha=0.1081^{\circ}$ which characterize the rigid body displacement. Hence, we are able to retrieve the deformable displacement field, see also Figure 9(a,b).

\subsubsection{Reconstruction with noisy data}

Next, we investigate how the reconstructions are influenced by noisy data. We assume that the given exact data $\left.\underline{\tilde{u}}\right|_{\Omega_{d}}=\underline{u}^{a n} \mid \Omega_{d}$ have been perturbed as:

$$
\begin{aligned}
& {\widetilde{u_{1}}}^{\varepsilon}(x)=u_{1}^{a n}(\underline{x})+v \times \delta \max \left(u_{1}^{a n}(\underline{x})\right) \rho, \underline{x} \in \Omega_{d}, \\
& {\widetilde{u_{2}}}^{\varepsilon}(\underline{x})=u_{2}^{a n}(\underline{x})+\delta \max \left(u_{2}^{a n}(\underline{x})\right) \rho, \underline{x} \in \Omega_{d}
\end{aligned}
$$

where $\delta$ is the level noise added to $\left.\underline{\widetilde{u}}\right|_{\Omega_{d}}=\underline{u}^{a n} \mid \Omega_{d}$ and $\rho$ is a pseudo-random number drawn from the standard uniform distribution in $[-1,1]$. In this way, the noise is proportional to each component of the displacement data.

The reconstructions of the components $u_{2}$ and $T_{2}$ on $\Gamma$, obtained with different noise levels $(1 \%, 3 \%, 5 \%$ and 10\%) are represented in Figure 11(a,b), respectively.

The reconstructions obtained are very accurate. Table 3 tabulates the results obtained for various levels of noise and various values of $c$, by specifying the number of iterations necessary to achieve convergence and the errors $u_{\text {error }}^{\Omega}, u_{\text {error }}^{\Gamma}$ and $T_{\text {error }}$. For each fixed noise level, the errors, for $\underline{u}$ 


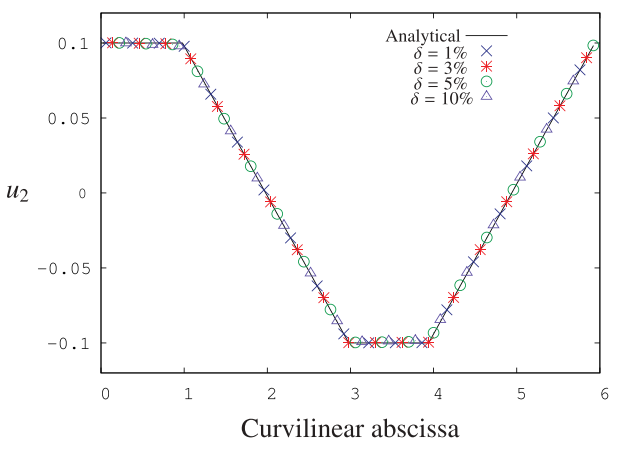

(a)

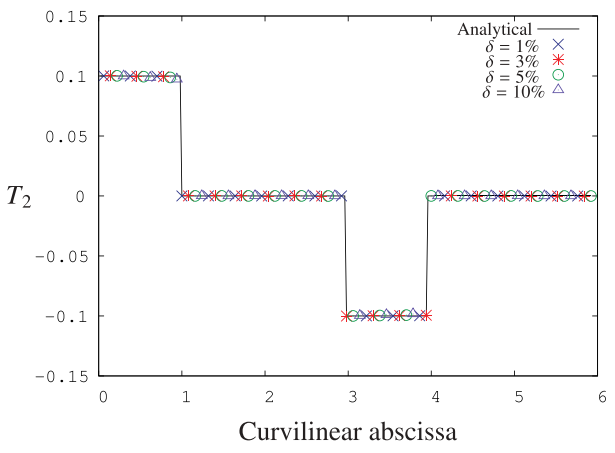

(b)

Figure 11. (a) Analytical, $u_{2}^{a n}$, and numerical, $u_{2}$, vertical displacements, and (b) analytical, $T_{2}^{a n}$, and numerical, $T_{2}$, vertical stress vector components, retrieved on $\Gamma$, for different noise levels and $c=1$.

and $\underline{T}$ respectively, remain fairly identical regardless of the value of the parameter $c$. This shows that even for noisy data, the numerical reconstructions are independent of $c$. Therefore, the combined fading regularization method-MFS algorithm is accurate and robust with respect to noisy data.

Figure $12(\mathrm{a}-\mathrm{c})$ shows the noisy vertical displacement data $u_{2}^{d}$ given in $\Omega_{d}$, the reconstruction of the vertical displacement component $u_{2}$ in $\Omega_{d}$ and the residual term $\left|u_{2}^{d}-u_{2}\right|$ in $\Omega_{d}$, respectively, obtained for data with $10 \%$ noise on $\left.\underline{\underline{u}}\right|_{\Omega_{d}}$ and $c=10^{-1}$. We retrieve a residual term (Figure 12(c)) which corresponds to the $10 \%$ added noise. This result shows that the proposed MFS-FRM algorithm allows for the denoising, i.e the filtering in a mechanical sense, of the data. This property is due to the definition of functional (12), which is composed of different terms with different roles. As in most inverse iterative methods, there are regularization terms which tend to zero as the number of iterations increases. However, in the present FRM algorithm, there is also a relaxation term that allows for the data blurred by noise to be taken into account. Therefore, we seek for a solution which is close to the data but does not exactly fits the data. In fact, the algorithm recomputes, at each step, a solution on the whole domain and not only a solution where the data are lacking.

\subsubsection{Influence of the parameter $\zeta$}

Next, we discuss the sensitivity of the numerical algorithm with respect to the size of the data grid $\Omega_{d}$, characterized by the parameter $\zeta$. We used noisy displacement field data which is generated for $\Omega_{d}=\Omega(\zeta=1)$ and, for each value of $\zeta$, we keep only the part of data which belongs to $\Omega_{d}$ and hence the algorithm works with comparable data, regardless the parameter $\zeta$. The errors $u_{\text {error }}^{\Omega}, u_{\text {error }}^{\Gamma}$ and $T_{\text {error }}$ are compared in Table 3. 


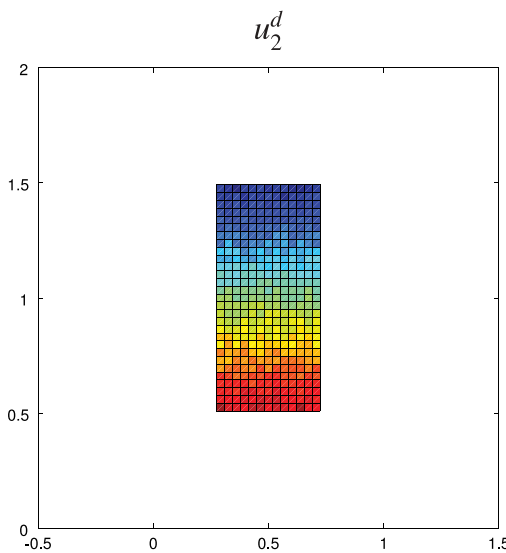

(a)

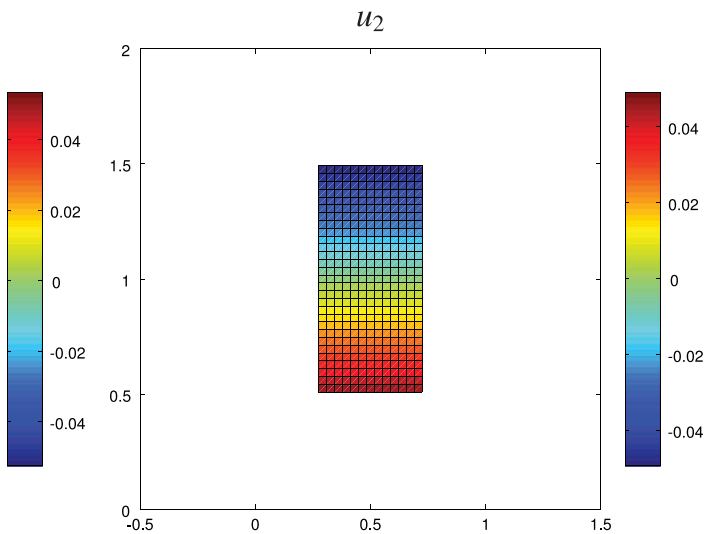

(b)

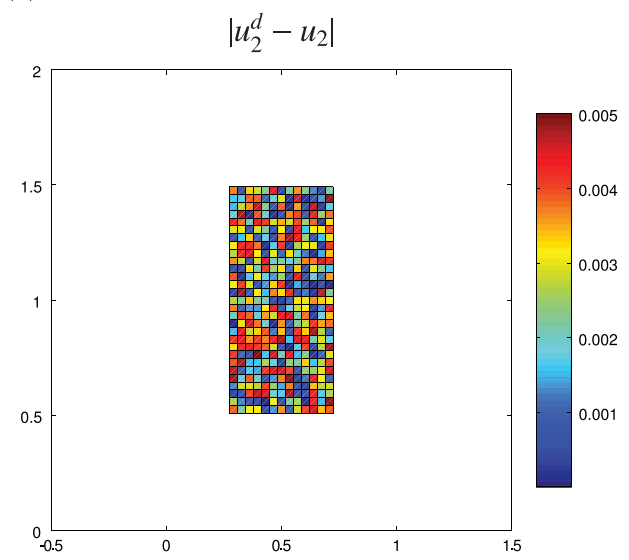

(c)

Figure 12. (a) Vertical displacement data, $u_{2}^{d}$, (b) reconstruction of the vertical displacement, $u_{2}$, in $\Omega_{d}$ and (c) residual term $\left|u_{2}^{d}-u_{2}\right|$ in $\Omega_{d}$, for $c=10^{-1}$ and $10 \%$ noise level.

Figure 13(a-c) shows the vertical displacement data, $u_{2}^{d}$, and the reconstructions of the vertical displacement $u_{2}$ in $\Omega_{d}$ and $u_{2}^{\Omega}$ in $\Omega$, obtained when applying the MFS-FRM algorithm and the stopping criterion introduced in Section 4.1.1.1. For these figures, the different values of the parameters are $\delta=5 \%, c=10^{-2}$ and $\zeta=\frac{1}{9}$.

Figure $14(\mathrm{a}, \mathrm{b})$ shows the reconstructions of the displacement components $u_{1}$ and $u_{2}$, on $\Gamma$, respectively, for various values of $\zeta$. It can be observed from these figures that the numerical solution are little affected by the size of the data grid. For some values of $\zeta$, the reconstructions are less accurate, however, they remain still good (see also Table 3 ). This is explained by the fact that the displacements are very small near the centre of $\Omega$, so that for small data grid $\left(\zeta \leq \frac{1}{9}\right)$ the noise component becomes larger than that of the displacements. 


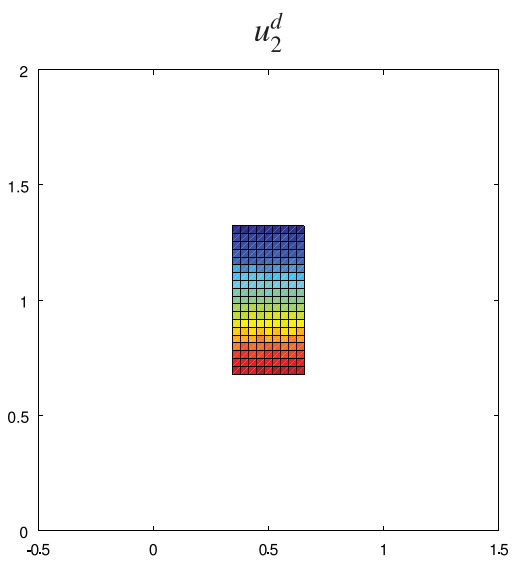

(a)

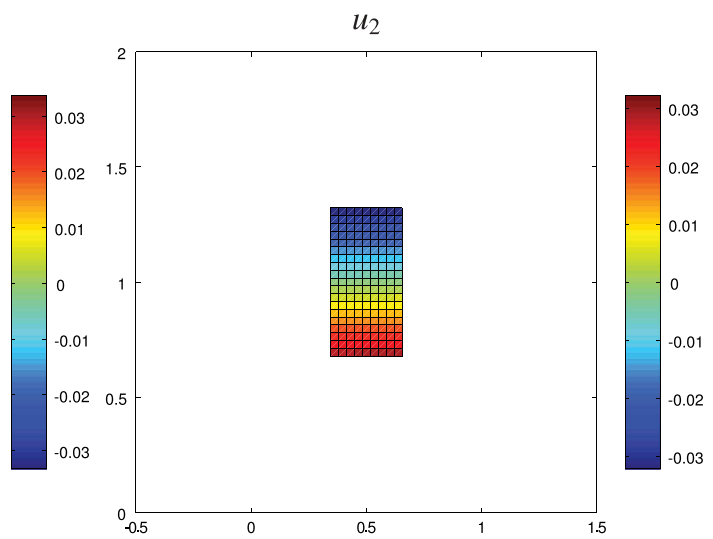

(b)

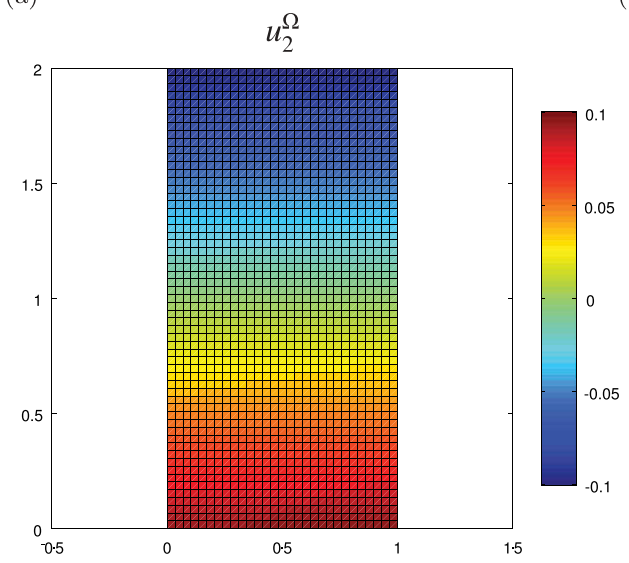

(c)

Figure 13. Known vertical displacement data $u_{2}^{d}$ (a) and reconstructed vertical displacement $u_{2}$ (b) in $\Omega_{d}$ and reconstructed vertical displacement $u_{2}^{\Omega}$ (c) in $\Omega$ for $\zeta=\frac{1}{9}, c=10^{-2}$ and $5 \%$ noise level.

\subsection{Unconfined compression test with non-uniform prescribed forces}

A similar study is further performed for the same analytical displacement field solution as in the first example, but with a different loading condition. The solution domain represented in Figure 15 is a subdomain of the initial one (Figure 2). This particular configuration allows one to apply the proposed algorithm to a geometry that causes a non-constant stress vector component $T_{2}$ on $\Gamma_{u}$.

The MFS parameters have been set as $N=90$ on $\tilde{\Gamma}=$ $\left\{\underline{x}=\left(x_{1}, x_{2}\right) \in \mathbb{R}^{2} \mid x_{1}^{2}+x_{2}^{2}=d^{2}\right\} \quad$ and $d=10$, whilst $M_{d}=80$ and $M_{u}=60$. The data grid $\Omega_{d}$ is defined by the parameter $\zeta=\frac{1}{4}$ and the domain $\Omega$ is discretized with 1552 points. 


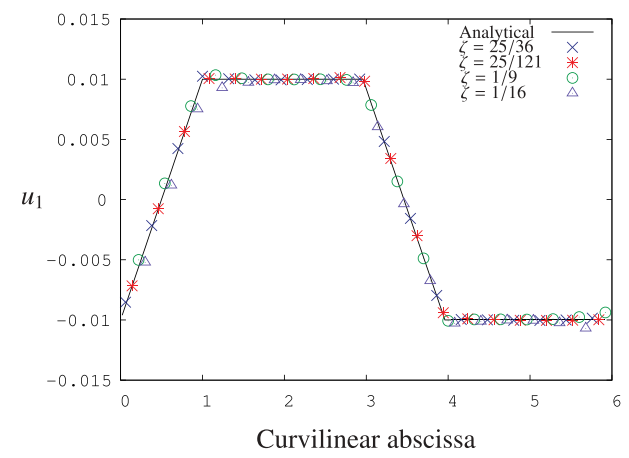

(a)

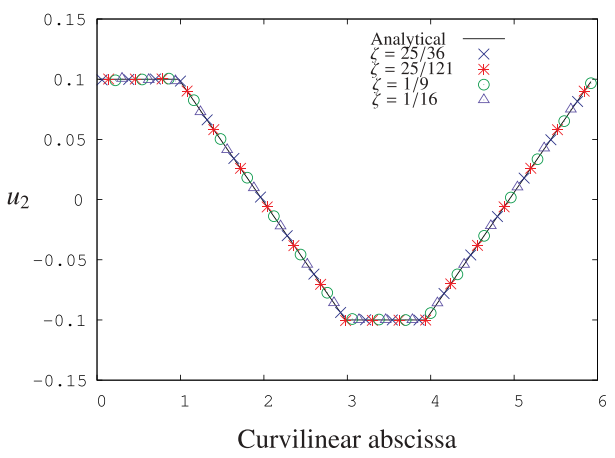

(b)

Figure 14. Analytical, $u_{1}^{a n}$, and numerical, $u_{1}$, displacements (a) and analytical, $u_{2}^{a n}$, and numerical, $u_{2}$, displacements (b) retrieved on $\Gamma$ for different parameters $\zeta, \delta=5 \%$, and $c=10^{-2}$.

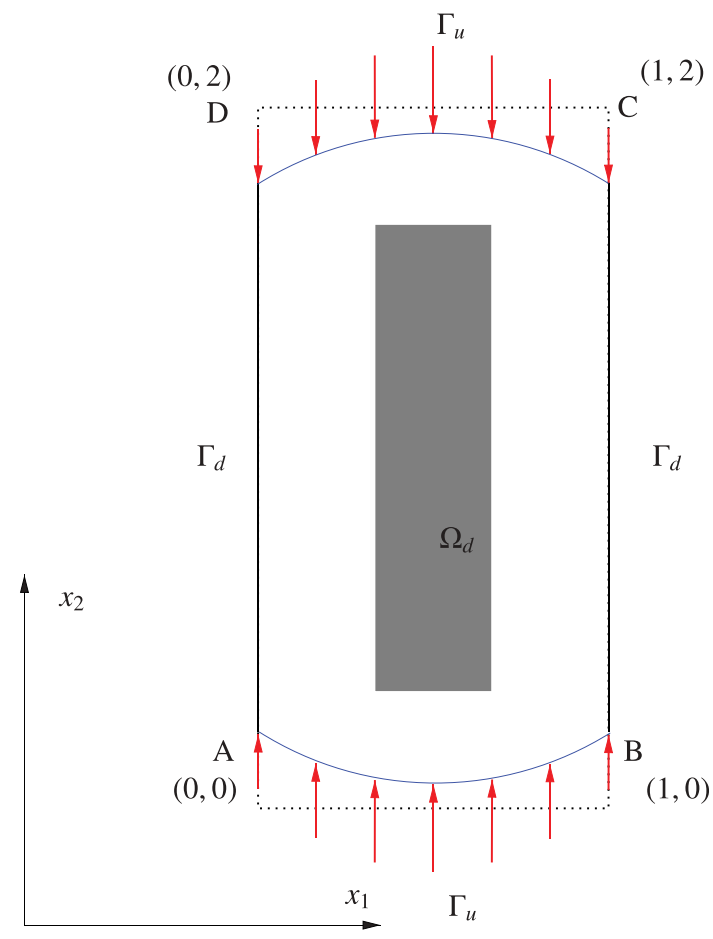

Figure 15. Compressed pavement with rounded shape.

Figure 16(a,b) shows the reconstructions of the displacement components $u_{1}$ and $u_{2}$ on $\Gamma$, respectively, and Figure $17(\mathrm{a}, \mathrm{b})$ shows the reconstructions of the stress vector components $T_{1}$ and $T_{2}$ on $\Gamma$, respectively, for a noise level of $1 \%, 3 \%, 5 \%$ and $10 \%$ and $c=10^{-1}$. In these figures the 


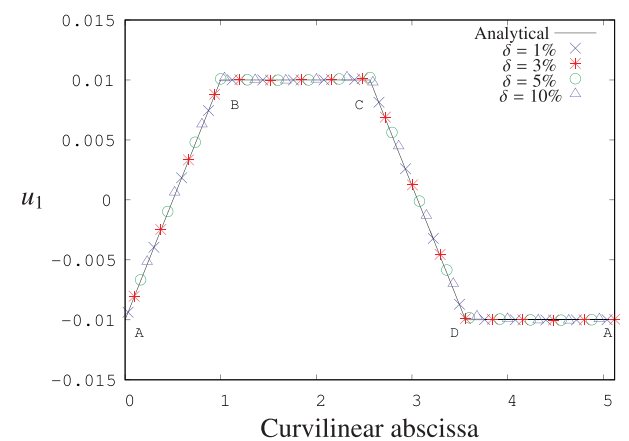

(a)

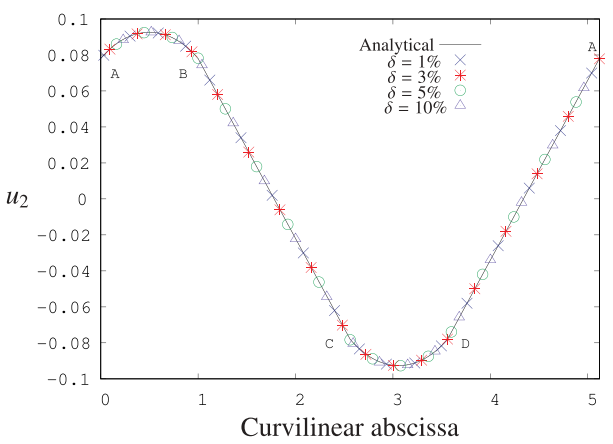

(b)

Figure 16. (a) Analytical, $u_{1}^{a n}$, and numerical $u_{1}$, horizontal displacements and (b) analytical, $u_{2}^{a n}$, and numerical $u_{2}$, vertical displacements, retrieved on $\Gamma$, for various noise levels and $c=10^{-1}$.

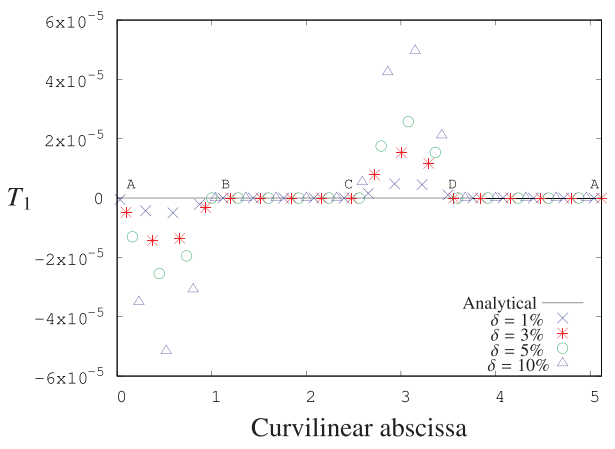

(a)

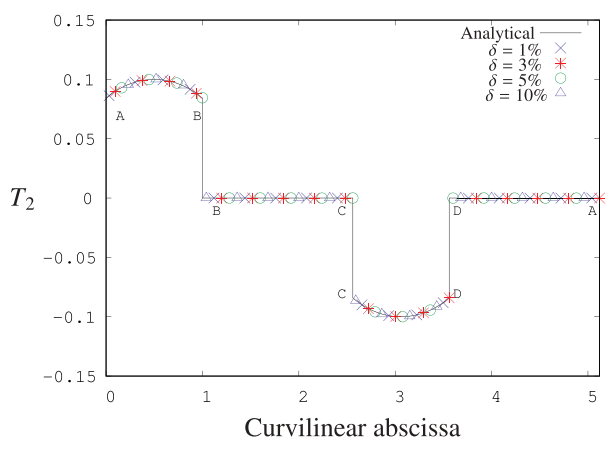

(b)

Figure 17. (a) Analytical, $T_{1}^{a n}$, and numerical, $T_{1}$, stress vector horizontal components, and (b) analytical, $T_{2}^{a n}$, and numerical, $T_{2}$, stress vector vertical components, retrieved on $\Gamma$ for various noise levels and $c=10^{-1}$.

results are displayed on the whole boundary $\Gamma$ thanks to the use of a curvilinear abscissa $s$, growing respectively from 0 to 1 as $M$ describes $\widehat{A B}$, from 1 to 2.56 as $M$ describes $\widehat{B C}$, from 2.56 to 3.56 as $M$ describes $\widehat{C D}$, and from 3.56 to 5.12 as $M$ describes $\widehat{D A}$ (Figure 15).

These curves reveal the accuracy of the reconstructions on $\Gamma$ as well as the ability of the fading regularization-MFS algorithm to deblur noisy data in $\Omega_{d}$ and to retrieve the displacement field in $\Omega$ (see also Figure 18(a,b)).

Table 4 summarizes, for various levels of noise, the number of iterations necessary to achieve convergence and the corresponding errors. It exhibits the same characteristics as those obtained for the previous example (Table 2). 


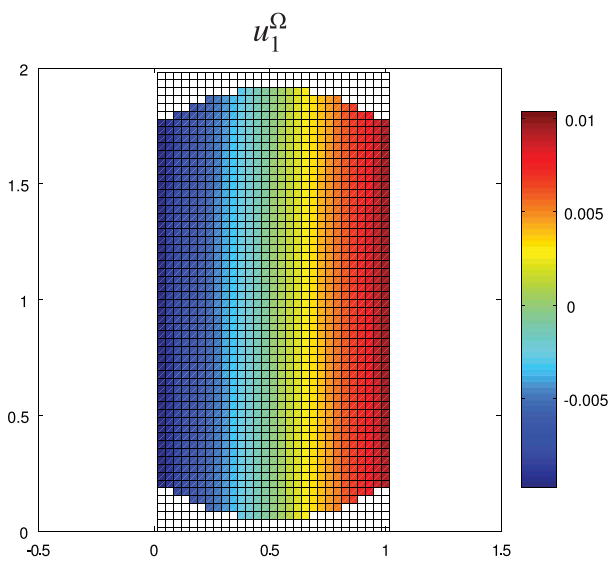

(a)

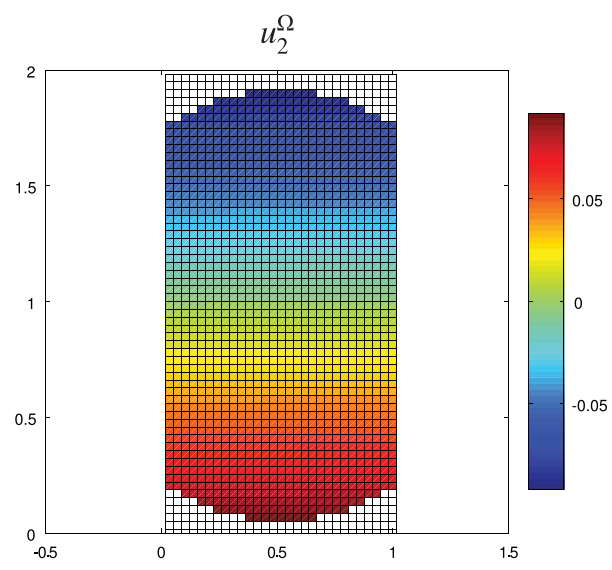

(b)

Figure 18. (a) The reconstruction of the horizontal displacement component, $u_{1}$, and (b) the reconstruction of the vertical displacement component, $u_{2}$, in $\Omega$ for $c=10^{-1}$ and a noise level $\delta=10 \%$.

Table 2. Influence of noise level $\delta$ and parameter $c$ on the number of iterations, $k$, required to achieve convergence, and on the errors $u_{\text {error }}^{\Omega} u_{\text {error }}^{\Gamma}$ and $T_{\text {error }}$.

\begin{tabular}{cllccc}
$\delta$ & \multicolumn{1}{c}{$k$} & \multicolumn{1}{c}{$k$} & $u_{\text {error }}^{\Omega}$ & $u_{\text {error }}^{\Gamma}$ & $T_{\text {error }}$ \\
\hline $1 \%$ & $10^{-2}$ & 33 & $2.87 \times 10^{-7}$ & $3.93 \times 10^{-7}$ & $1.10 \times 10^{-7}$ \\
& $10^{-1}$ & 187 & $2.87 \times 10^{-7}$ & $3.93 \times 10^{-7}$ & $1.10 \times 10^{-7}$ \\
& 10 & 3008 & $2.51 \times 10^{-7}$ & $3.33 \times 10^{-7}$ & $9.49 \times 10^{-8}$ \\
$3 \%$ & $10^{-2}$ & 35 & $2.69 \times 10^{-6}$ & $3.53 \times 10^{-6}$ & $9.91 \times 10^{-7}$ \\
& $10^{-1}$ & 199 & $2.59 \times 10^{-6}$ & $3.53 \times 10^{-6}$ & $9.91 \times 10^{-7}$ \\
& 10 & 3924 & $2.42 \times 10^{-6}$ & $3.27 \times 10^{-6}$ & $9.24 \times 10^{-7}$ \\
$5 \%$ & $10^{-2}$ & 37 & $7.18 \times 10^{-6}$ & $9.82 \times 10^{-6}$ & $2.75 \times 10^{-6}$ \\
& $10^{-1}$ & 204 & $7.19 \times 10^{-6}$ & $9.82 \times 10^{-6}$ & $2.75 \times 10^{-6}$ \\
& 10 & 4572 & $6.92 \times 10^{-6}$ & $9.37 \times 10^{-6}$ & $2.64 \times 10^{-6}$ \\
$10 \%$ & $10^{-2}$ & 37 & $2.87 \times 10^{-5}$ & $3.93 \times 10^{-5}$ & $1.10 \times 10^{-5}$ \\
& $10^{-1}$ & 216 & $2.87 \times 10^{-5}$ & $3.93 \times 10^{-5}$ & $1.10 \times 10^{-5}$ \\
& 10 & 5495 & $2.82 \times 10^{-5}$ & $3.84 \times 10^{-5}$ & $1.08 \times 10^{-5}$ \\
\hline
\end{tabular}

Table 3. Influence of the data grid size (parameter $\zeta$ ) on the number of iterations, $k$, required to achieve convergence, and on the errors $u_{e r r o r}^{\Omega} u_{e r r o r}^{\Gamma}$ and $T_{\text {error }}$.

\begin{tabular}{llccc}
\hline$\zeta$ & $k$ & $u_{\text {error }}^{\Omega}$ & $u_{\text {error }}^{\Gamma}$ & $T_{\text {error }}$ \\
\hline$\frac{25}{36}$ & 10 & $7.51 \times 10^{-6}$ & $1.26 \times 10^{-5}$ & $1.35 \times 10^{-5}$ \\
$\frac{4}{9}$ & 13 & $1.50 \times 10^{-5}$ & $2.51 \times 10^{-5}$ & $2.88 \times 10^{-5}$ \\
$\frac{1}{4}$ & 37 & $7.19 \times 10^{-6}$ & $9.82 \times 10^{-6}$ & $2.75 \times 10^{-6}$ \\
$\frac{25}{121}$ & 49 & $7.86 \times 10^{-6}$ & $8.54 \times 10^{-6}$ & $4.98 \times 10^{-6}$ \\
$\frac{4}{25}$ & 86 & $6.66 \times 10^{-6}$ & $9.23 \times 10^{-6}$ & $8.86 \times 10^{-6}$ \\
$\frac{1}{9}$ & 210 & $4.38 \times 10^{-5}$ & $6.42 \times 10^{-5}$ & $6.32 \times 10^{-5}$ \\
$\frac{1}{16}$ & 1205 & $9.73 \times 10^{-5}$ & $1.44 \times 10^{-4}$ & $5.65 \times 10^{-5}$ \\
\hline
\end{tabular}


Table 4. Influence of the noise level, $\delta$, on the number of iterations, $k$, required

\begin{tabular}{ccccc}
\multicolumn{5}{c}{ to achieve convergence and on the norms $u_{\text {error }}^{\Omega} u_{\text {error }}^{\Gamma}$ and $T_{\text {error }}$ for $c=10^{-1}$. } \\
\hline$\delta$ & $k$ & $u_{\text {error }}^{\Omega}$ & $u_{\text {error }}^{\Gamma}$ & $T_{\text {error }}$ \\
\hline $0 \%$ & 51 & $3.80 \times 10^{-20}$ & $7.70 \times 10^{-20}$ & $1.23 \times 10^{-18}$ \\
$1 \%$ & 99 & $2.55 \times 10^{-7}$ & $3.33 \times 10^{-7}$ & $1.09 \times 10^{-7}$ \\
$3 \%$ & 109 & $2.30 \times 10^{-6}$ & $3.00 \times 10^{-6}$ & $9.78 \times 10^{-7}$ \\
$5 \%$ & 112 & $6.38 \times 10^{-6}$ & $8.33 \times 10^{-6}$ & $2.72 \times 10^{-6}$ \\
$10 \%$ & 120 & $2.55 \times 10^{-5}$ & $3.33 \times 10^{-5}$ & $1.09 \times 10^{-5}$ \\
\hline
\end{tabular}

\section{Application of the MFS-FRM algorithm to the analysis of a diametral compression test}

The displacement fields at the surface of solids can be evaluated using full-field kinematic measurements (Sutton, McNeill, Helm, \& Chao, 2000). The main techniques for tracking motion and deformation are photo-elasticity, Moiré, holographic and speckle interferometry, grid method and digital image correlation (DIC). DIC was proposed at the beginning of the 1980s (Burt et al., 1982, Sutton, Wolters, Peters, Ranson, \& McNeill, 1983). The principle of DIC is to compare two recorded images and determine the displacement field that optimizes a cross-correlation. A random speckle pattern is applied on the surface to measure the displacement field based on the assumption that the surface texture passively follows the displacement of the analyzed solid. The matching in greyscale intensity values is realised by zones of interest which are small windows of the considered domain of interest. This type of approach will be referred to as local (Hild \& Roux, 2012).

The objective now is to apply the proposed algorithm to experimental displacement fields measured by DIC. We are interested in a particular contact problem, already analysed numerically by Delvare et al. (Delvare et al., 2010). In this paper, we deal with a diametral compression test on a polyethylene cylinder. This case was selected because we face several common characteristic issues: the displacement field cannot be measured near the contact area and is affected by noise, the extension of the contact area and the pressure distribution are unknown, and a rigid body motion may appear at the beginning of the test.

\subsection{Experimental set-up}

A polyethylene cylinder $(v=0.46)$ is compressed between two parallel rigid plane supports, see Figure 19(a). The cylinder has a radius $R=61.6$ $\mathrm{mm}$ and a length $e=2 \times R$. The compression load is generated by an imposed vertical displacement $\underline{u}_{0}$ of the upper hardened steel loading plate while the lower support remains motionless.

A Photron Fastcam SA5 camera, with a frame rate of 7000 images/ second and a $1024 \times 1024$ pixels resolution, is used to record images in 


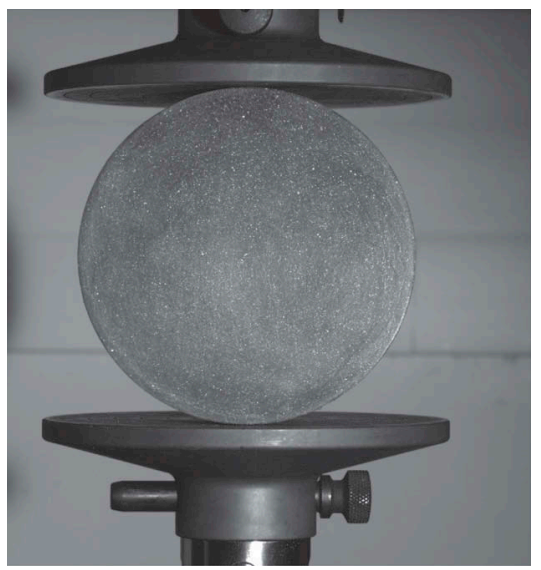

(a)

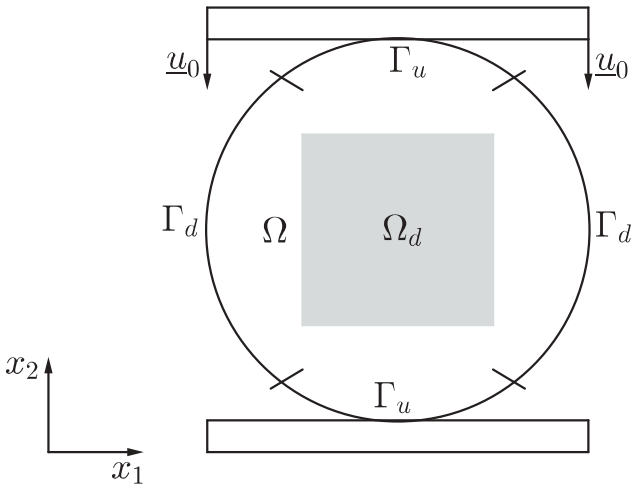

(b)

Figure 19. (a) Compressed polyethylene cylinder (b) 2D modelling.

digital form. In order to obtain images having a good distribution of greyscale, the surface of the cylinder is prepared beforehand with the realization of a speckle. The two-dimensional displacement fields in $\Omega_{d}$ are provided from DIC using Icasoft software (Touchal, Morestin, \& Brunet, 1996).

\subsection{MFS-FRM post-processing and results}

Due to the thickness of the cylinder, a two-dimensional study with a plane strain assumption is performed (Figure 19(b)). To avoid a bias due to the initial mechanical slack adjustment, the analysis is performed between images 500 and 2000 for which a force transducer measures an intensity force equal to $15120 \mathrm{~N}$ on the top plate. Due to the DIC, the displacement field is known in a central part of the specimen $\Omega_{d}$ and the stress vectors are considered to be equal to zero on the free-force edges $\Gamma_{d}$, see Figure 19 (b). The real sizes of $\Gamma_{u}$ and $\Gamma_{d}$ remain unknown. To fully define the mechanical problem, the $\Gamma_{u}$ extent is chosen greater than the real one and is set to $\beta=\frac{\pi}{20}$.

Note that $\Gamma=\Gamma_{d} \cup \Gamma_{u}$ with $\Gamma_{d}=\left\{\underline{x} \in \Gamma \mid 0 \leq \theta(\underline{x}) \leq \frac{\pi}{2}-\frac{\beta}{2}\right\} \cup\left\{\underline{x} \in \Gamma \mid \frac{\pi}{2}+\right.$ $\left.\frac{\beta}{2} \leq \theta(\underline{x}) \leq \frac{3 \pi}{2}-\frac{\beta}{2}\right\} \cup\left\{\underline{x} \in \Gamma \mid \frac{3 \pi}{2}+\frac{\beta}{2} \leq \theta(\underline{x}) \leq 2 \pi\right\} \quad$ and $\Gamma_{u}=\left\{\underline{x} \in \Gamma \mid \frac{\pi}{2}-\frac{\beta}{2}\right.$ $\left.<\theta(\underline{x})<\frac{\pi}{2}+\frac{\beta}{2}\right\} \cup\left\{\underline{x} \in \Gamma \mid \frac{3 \pi}{2}-\frac{\beta}{2}<\theta(\underline{x})<\frac{3 \pi}{2}+\frac{\beta}{2}\right\}$, where $\theta(\underline{x})$ is the angular polar coordinate of $\underline{x}$.

We present the reconstruction of the displacement fields in the whole domain $\Omega$ and the identification of the missing boundary conditions computed by applying the proposed MFS-FRM algorithm presented in Section 3 to displacement fields measured in $\Omega_{d}$. The analysis is performed 


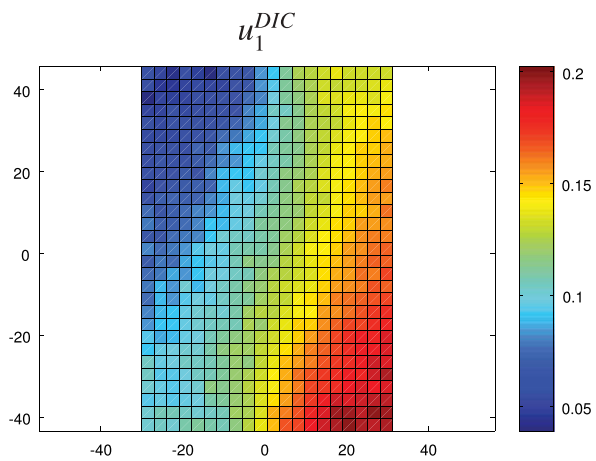

(a)

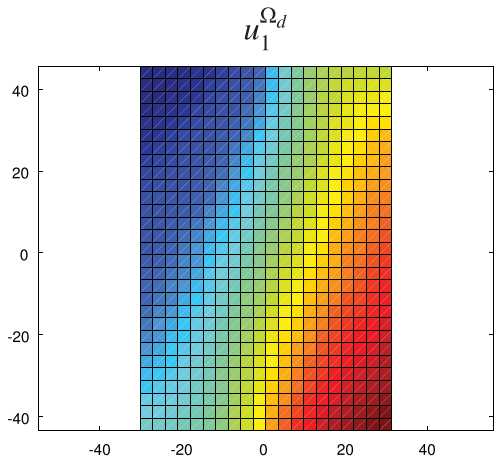

(c)

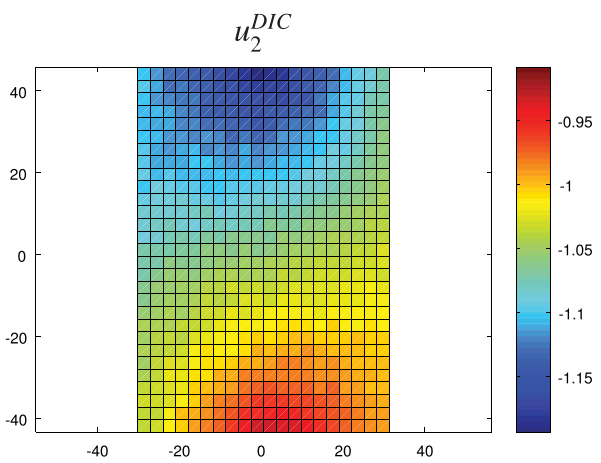

(b)

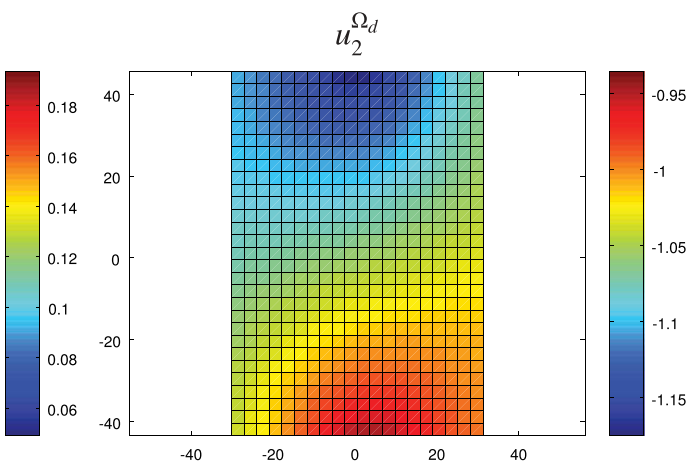

(d)

Figure 20. DIC experimental (a) horizontal displacement component $u_{1}^{D / C}$, and (b) vertical displacement component $u_{2}^{D / C}$, and FRM-MFS reconstructed (c) horizontal displacement component $u_{1}^{\Omega_{d}}$, and (d) vertical displacement component $u_{2}^{\Omega_{d}}$, in $\Omega_{d}$.

in two steps. Firstly, a dimensionless problem (i.e. $E=1 \mathrm{~N} . \mathrm{m}^{-2}$ ) is analyzed and, secondly, the Young's modulus is identified.

The corresponding MFS parameters have been set as $N=120$ on $\tilde{\Gamma}=$ $\left\{\underline{x}=\left(x_{1}, x_{2}\right) \in \mathbb{R}^{2} \mid x_{1}^{2}+x_{2}^{2}=d^{2}\right\} \quad$ and $d=65$, whilst $M_{d}=120$ and $M_{u}=40$. The domain $\Omega_{d}$ is defined by its 630 points defined by $\left(x_{d}, y_{d}\right)$ such that $-30.1 \mathrm{~mm}<x_{d}<31.3 \mathrm{~mm}$ and $-43.5 \mathrm{~mm}<y_{d}<45.6 \mathrm{~mm}$, which corresponds to a parameter $\zeta \approx \frac{1}{2}$.

Figure 20(a,c) represents, in $\Omega_{d}$, the displacement component $u_{1}^{D I C}$, obtained by DIC and the reconstruction $u_{1}^{\Omega_{d}}$ obtained when applying the FRM-MFS algorithm and using the stopping criterion introduced in Section 4.1.1.1 and $c=10^{-1}$, respectively. Figure $20(b, d)$ shows the same quantities for the vertical displacement component $u_{2}$.

Figure 20(a,b) highlights the presence of a rigid body motion during the test. It can be remarked from the comparison of the numerical results against experimental ones, the accuracy of the reconstructions in $\Omega_{d}$ and 


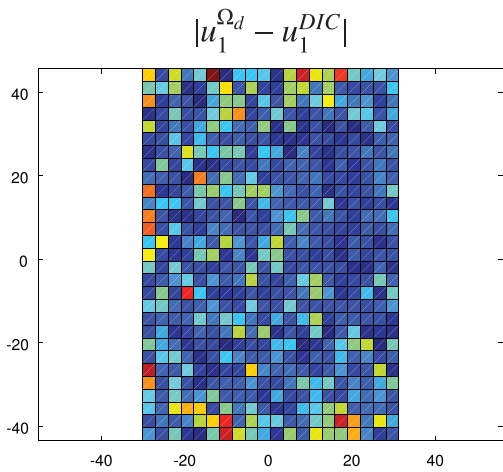

(a)

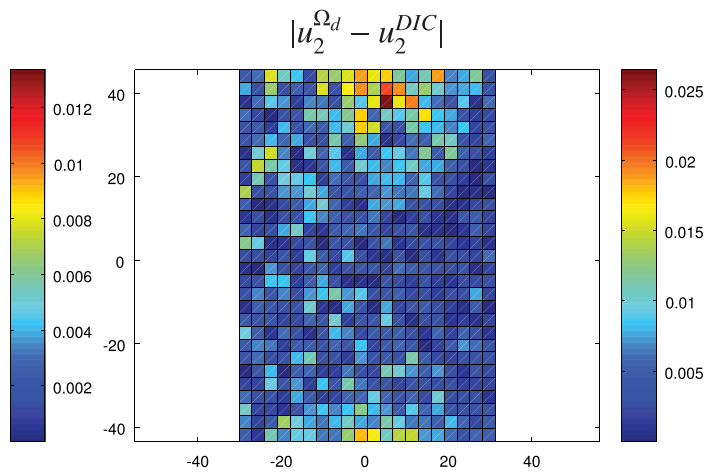

(b)

Figure 21. Residual components, (a) $\left|u_{1}^{\Omega_{d}}-u_{1}^{D / C}\right|$, and (b) $\left|u_{2}^{\Omega_{d}}-u_{2}^{D / C}\right|$, in $\Omega_{d}$.

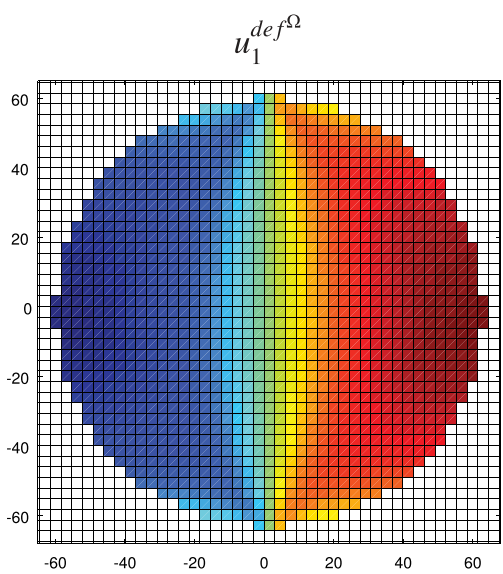

(a)

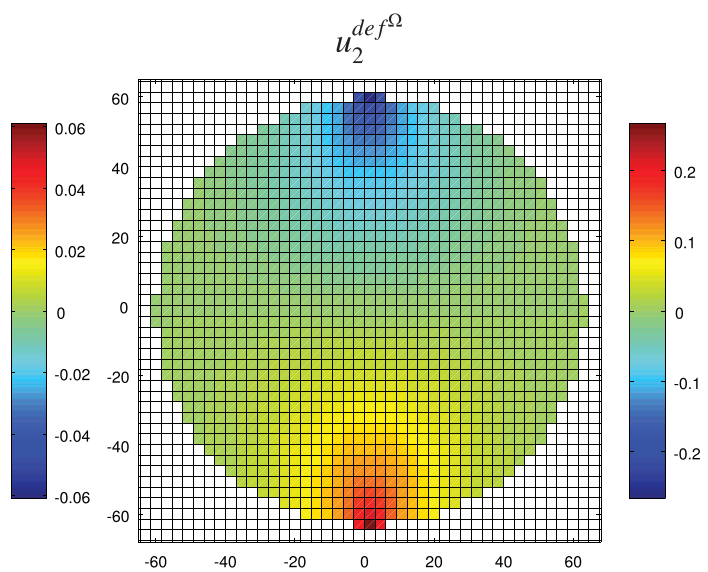

(b)

Figure 22. Reconstruction of the deformable displacement components, (a) $u_{1}^{\text {def }}$, and (b) $u_{2}^{\text {def }^{\Omega}}$, in $\Omega$.

the capability of the FRM-MFS algorithm to deblur the noisy data in $\Omega_{d}$. The noise level can be estimated by analyzing the residual terms $\left|u_{1}^{\Omega_{d}}-u_{1}^{D I C}\right|$ and $\left|u_{2}^{\Omega_{d}}-u_{2}^{D I C}\right|$, respectively, shown in Figure 21 . It is found to be about $10 \%$ for $u_{1}$ and $u_{2}$.

With a post-processing, using a non-linear optimization procedure, we are able to eliminate the rigid body displacement component. According to the notations used in Section 4.1.2, we identify the following parameters which characterize the rigid body displacement to be $a=0.1212, b=-1.0509$ and $\alpha=0.0405^{\circ}$. The deformable displacement components, $u_{1}^{d e f^{\Omega}}$ and $u_{2}^{d e f^{\Omega}}$, are represented in the whole specimen in Figure 22(a,b), respectively.

Figure 23(a,b) shows the reconstruction of the displacement boundary conditions on $\Gamma$. Figure 24(a,b) shows the reconstruction of $T_{1}$ on $\Gamma$ and the reconstruction of $T_{2}$ near the upper contact zone, respectively. 


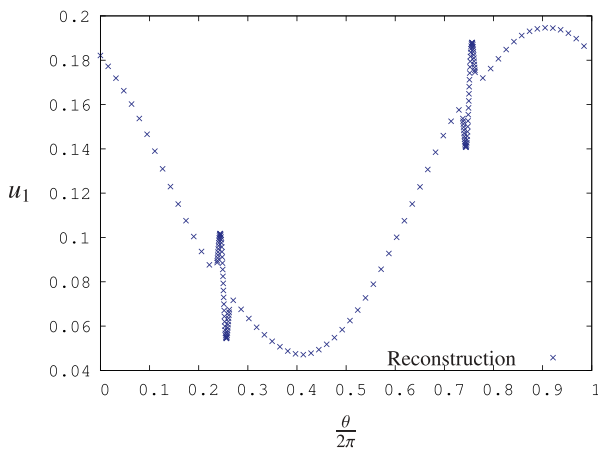

(a)

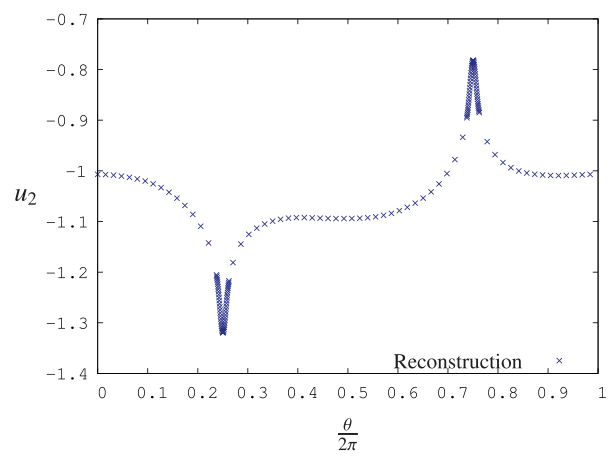

(b)

Figure 23. Numerical components (a) $u_{1}$, and (b) $u_{2}$, of the boundary displacement conditions retrieved on $\Gamma$.

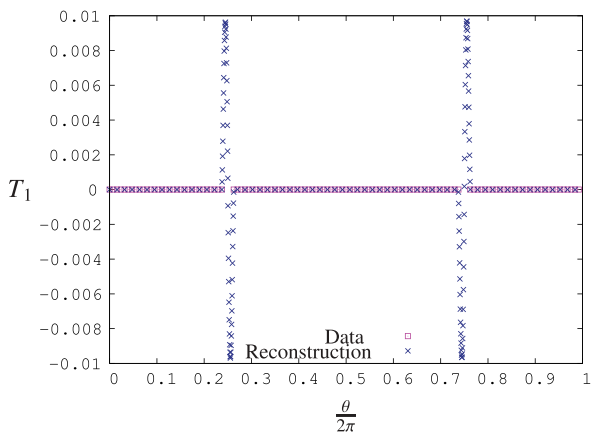

(a)

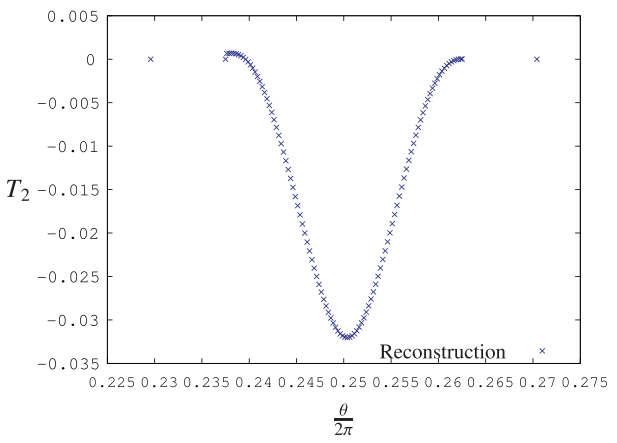

(b)

Figure 24. (a) Numerical stress vector horizontal component $T_{1}$ retrieved on $\Gamma$ and (b) a zoom on the stress vector vertical component $T_{2}$ retrieved near the upper contact area (upper part of $\Gamma_{u}$ ).

The reconstructions of the displacements and the stress vectors, obtained on $\Gamma$, are accurate and allow to identify a posteriori the extension of the contact zones. Due to the reconstruction of $T_{2}$ near the upper contact area, we identify the Young's modulus to be $E=907 \mathrm{MPa}$ by connecting the contact pressure distribution to the force applied to the top plate.

Due to the discretization using the MFS, we can compute by a postprocessing the analytical deformations in the entire domain $\Omega$. Two strain components, $\varepsilon_{11}$ and $\varepsilon_{22}$, are represented in Figure 25(a,b), respectively.

\section{Conclusion}

The aim of this article is to present a method for solving an inverse problem in isotropic and two-dimensional linear elasticity. This inverse problem consists of, from the knowledge of the displacement field on a part of the sample and of the stress vector on a part of the boundary, 


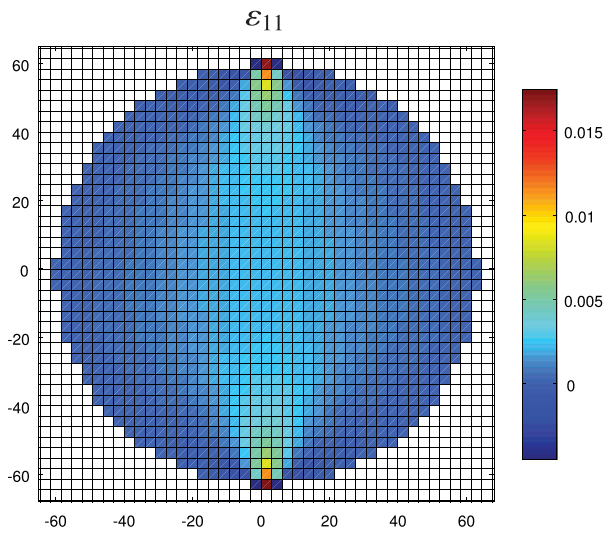

(a)

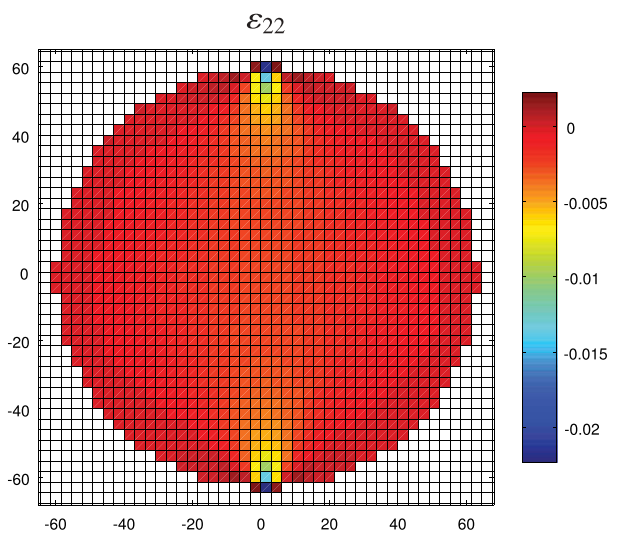

(b)

Figure 25. Numerical strain components, (a) $\varepsilon_{11}$, and (b) $\varepsilon_{22}$, retrieved in $\Omega$.

finding the isotropic linear elastic solution over the entire domain, as well as the boundary conditions, that are inaccessible to measurements. The proposed method is based on a fading regularization algorithm and is numerically implemented using the MFS.

This approach reduces the resolution of the inverse problem to an iterative process composed of a sequence of optimization problems, where the functional to be minimized contains two terms. The first one is a relaxation term which represents, on the part of the domain where the displacement field is available/measured, the difference between the optimal displacements (solution of the Lamé equation) and the measurements. The second term is a regularization term, which tends to zero as the iterative process continues. It represents the difference between two successive optimal elements. Several examples, first using synthetic data and then using experimental DIC data, revealed the accuracy, convergence, stability and efficiency of the proposed inverse method, and its ability to denoise noisy measurements and to manage parasite rigid body displacements. The use of the MFS also allows us to analytically compute the components of the strain tensor over the entire domain.

For future works, we plan, on one hand, to implement the fading regularization algorithm using the finite element method and on the other hand, to apply the current method for other materials in order to show the ability of the method to detect anelastic or microscopic phenomena.

\section{Note}

1. The reconstructions of the horizontal component of displacement $u_{1}$ and the horizontal component of the stress vector $T_{1}$ are not presented because the results are similar to those obtained for the $u_{2}$ and $T_{2}$. 


\section{Acknowledgements}

The authors would like to thank French Region Centre Val de Loire (in the framework of IMFRA2 APR-2012) and French Region Normandie for supporting this project.

\section{Disclosure statement}

No potential conflict of interest was reported by the authors.

\section{Funding}

This work was supported by French Region Centre Val de Loire [IMFRA2 APR-2012] and French Region Normandie.

\section{ORCID}

J-L. Hanus (D) http://orcid.org/0000-0001-7325-2178

F. Delvare (D) http://orcid.org/0000-0002-4750-8499

\section{References}

Andrieux, S., \& Baranger, T. N. (2008). An energy error-based method for the resolution of the Cauchy problem in 3D linear elasticity. Computer Methods in Applied Mechanics and Engineering, 197(9), 902-920.

Arai, M., Nishida, T., \& Adachi, T. (2000). Identification of dynamic pressure distribution applied to the elastic thin plate. In M. Tanaka, \& G. S. Dulikravich (Eds.), Inverse problems in Engineering Mechanics II (Int sym inverse problems in eng mech 2000, Nagano, Japan) (pp. 129-138) Elsevier Science Ltd. ISBN 9780080436937. doi: 10.1016/ B978-008043693-7/50086-6.

Baranger, T. N., \& Andrieux, S. (2008). An optimization approach for the Cauchy problem in linear elasticity. Structural and Multidisciplinary Optimization, 35(2), 141-152.

Berger, J. R., \& Karageorghis, A. (2001). The method of fundamental solutions for layered elastic materials. Engineering Analysis with Boundary Elements, 25(10), 877-886.

Burt, P. J., Yen, C., \& Xu, X. (1982). Local correlation measures for motion analysis: A comparative study. Proc. IEEE Conf. on Pattern Recognition and Image Processing (pp. 269-274). Washington, DC.

Caillé, L., Delvare, F., Marin, L., \& Michaux-Leblond, N. (2017). Fading regularization MFS algorithm for the Cauchy problem associated with the two-dimensional Helmholtz equation. International Journal of Solids and Structures, 125, 122-133.

Cimetière, A., Delvare, F., Jaoua, M., \& Pons, F. (2001). Solution of the Cauchy problem using iterated Tikhonov regularization. Inverse Problems, 17(3), 553.

Cimetière, A., Delvare, F., Jaoua, M., \& Pons, F. (2002). An inversion method for harmonic functions reconstruction. International Journal of Thermal Sciences, 41(6), 509-516.

Cimetière, A., Delvare, F., \& Pons, F. (2000). Une méthode inverse à régularisation évanescente [An inverse method with vanishing regularization]. Comptes Rendus De l'Académie Des Sciences-Series IIB-Mechanics, 328(9), 639-644. 
Delvare, F., Cimetière, A., Hanus, J.-L., \& Bailly, P. (2010). An iterative method for the Cauchy problem in linear elasticity with fading regularization effect. Computer Methods in Applied Mechanics and Engineering, 199(49), 3336-3344.

Delvare, F., Cimetière, A., \& Pons, F. (2002). An iterative boundary element method for Cauchy inverse problems. Computational Mechanics, 28(3-4), 291-302.

Dennis, B. H., Dulikravich, G. S., \& Yoshimura, S. (2004). A finite element formulation for the determination of unknown boundary conditions for three-dimensional steady thermoelastic problems. Journal of Heat Transfer, 126(1), 110-118.

Durand, B., Delvare, F., \& Bailly, P. (2011). Numerical solution of Cauchy problems in linear elasticity in axisymmetric situations. International Journal of Solids and Structures, 48(21), 3041-3053.

Grédiac, M., Hild, F., \& Pineau, A. (Eds.) (2012). Full-field measurements and identification in solid mechanics. London: ISTE; Hoboken, N.J.: Wiley. ISBN: 9781118578469. doi: $10.1002 / 9781118578469$

Hadamard, J. (1923). Lectures on Cauchy's problem in linear partial differential equations. New Haven: Yale University Press.

Hild, F., \& Roux, S. (2012). Comparison of local and global approaches to digital image correlation. Experimental Mechanics, 52(9), 1503-1519.

Koya, T., Yeih, W., \& Mura, T. (1993). An inverse problem in elasticity with partially overprescribed boundary conditions, Part II: Numerical details. Journal of Applied Mechanics, 60(3), 601-606.

Maniatty, A., Zabaras, N., \& Stelson, K. (1989). Finite element analysis of some inverse elasticity problems. Journal of Engineering Mechanics, 115(6), 1303-1317.

Marin, L., Delvare, F., \& Cimetière, A. (2016). Fading regularization MFS algorithm for inverse boundary value problems in two-dimensional linear elasticity. International Journal of Solids and Structures, 78-79, 9-20.

Marin, L., Elliott, L., Ingham, D. B., \& Lesnic, D. (2001). Boundary element method for the Cauchy problem in linear elasticity. Engineering Analysis with Boundary Elements, 25(9), 783-793.

Marin, L., Hào, D. N., \& Lesnic, D. (2002). Conjugate gradient-Boundary element method for the Cauchy problem in elasticity. The Quarterly Journal of Mechanics and Applied Mathematics, 55(2), 227-247.

Marin, L., \& Lesnic, D. (2002). Regularized boundary element solution for an inverse boundary value problem in linear elasticity. Communications in Numerical Methods in Engineering, 18(11), 817-825.

Marin, L., \& Lesnic, D. (2003). BEM first-order regularisation method in linear elasticity for boundary identification. Computer Methods in Applied Mechanics and Engineering, 192(16), 2059-2071.

Marin, L., \& Lesnic, D. (2004). The method of fundamental solutions for the Cauchy problem in two-dimensional linear elasticity. International Journal of Solids and Structures, 41(13), 3425-3438.

Marin, L., \& Lesnic, D. (2005). Boundary element-Landweber method for the Cauchy problem in linear elasticity. IMA Journal of Applied Mathematics, 70(2), 323-340.

Sutton, M. A., McNeill, S. R., Helm, J. D., \& Chao, Y. J. (2000). Advances in twodimensional and three-dimensional computer vision. In P. K. Rastogi (Ed.), Photomechanics. Topics in Applied Physics, vol 77 (pp. 323-372). Heidelberg, Berlin: Springer. doi: 10.1007/3-540-48800-6_10

Sutton, M. A., Orteu, J. J., \& Schreier, H. (2009). Image correlation for shape, motion and deformation measurements: Basic concepts, theory and applications. New York, NY: 
Springer Science \& Business Media. ISBN 978-0-387-78746-6. doi: 10.1007/978-0-38778747-3.

Sutton, M. A., Wolters, W. J., Peters, W. H., Ranson, W. F., \& McNeill, S. R. (1983). Determination of displacements using an improved digital correlation method. Image and Vision Computing, 1(3), 133-139.

Tikhonov, A. N., \& Arsenin, V. Y. (1977). Solution of ill-posed problems. New York, NY: John Wiley and Sons, Inc.; Washington, D.C: V. H. Winston \& Sons.

Touchal, S., Morestin, F., \& Brunet, M. (1996). Mesure de champs de déplacements et de déformations par corrélation d'images numériques. In Cailletaud (Ed.), Proc. Actes du Colloque National Mécamat'96 (pp. 179-182). Aussois.

Yeih, W., Koya, T., \& Mura, T. (1993). An inverse problem in elasticity with partially overprescribedboundary conditions, Part I: Theoretical approach. Journal of Applied Mechanics, 60(3), 595-600. 